\title{
Establishment of a Bernard-Soulier syndrome model in zebrafish
}

Qing Lin, ${ }^{1,2^{\star}}$ Riyang Zhou, ${ }^{1,2^{\star}}$ Panpan Meng, ${ }^{2 \star}$ Liangliang Wu, ${ }^{2}$ Lian Yang, ${ }^{2}$ Wenyu Liu, ${ }^{2}$ Jiaye Wu, ${ }^{2}$ Yuhuan Cheng, ${ }^{1}$ Linjuan Shi ${ }^{1}$ and Yiyue Zhang ${ }^{1,2}$

${ }^{1}$ Key Laboratory of Zebrafish Modeling and Drug Screening for Human Diseases of Guangdong Higher Education Institutes, Department of Developmental Biology, School of Basic Medical Sciences, Southern Medical University and ${ }^{2}$ Division of Cell, Developmental and Integrative Biology, School of Medicine, South China University of Technology, Guangzhou, China

${ }^{*} Q L, R Z$ and $P M$ contributed equally as co-first authors.

\section{Correspondence:}

Yiyue Zhang

mczhangyy@scut.edu.cn

Received: $\quad$ March 30, 2021

Accepted: $\quad$ August 6, 2021.

Prepublished: August 19, 2021.

https://doi.org/10.3324/haematol.2021.278893

(92022 Ferrata Storti Foundation

Haematologica material is published under a CC $\mathrm{BY}-\mathrm{NC}$ license @() $\Theta$

\begin{abstract}
Platelets play an essential role in thrombosis and hemostasis. Abnormal hemostasis can cause spontaneous or severe post-traumatic bleeding. Bernard-Soulier syndrome (BSS) is a rare inherited bleeding disorder caused by a complete quantitative deficiency in the GPIb-IX-V complex. Multiple mutations in GP9 lead to the clinical manifestations of BSS. Understanding the roles and underlying mechanisms of GP9 in thrombopoiesis and establishing a proper animal model of BSS would be valuable to understand the disease pathogenesis and to improve its medical management. Here, by using CRISPR-Cas9 technology, we created a zebrafish gp9sMu15 mutant to model human BSS. Disruption of zebrafish gp9 led to thrombocytopenia and a pronounced bleeding tendency, as well as an abnormal expansion of progenitor cells. The gp9sMu15 zebrafish can be used as a BSS animal model as the roles of GP9 in thrombocytopoiesis are highly conserved from zebrafish to mammals. Utilizing the BSS model, we verified the clinical GP9 mutations by in vivo functional assay and tested clinical drugs for their ability to increase platelets. Thus, the inherited BSS zebrafish model could be of benefit for in vivo verification of patient-derived GP9 variants of uncertain significance and for the development of potential therapeutic strategies for BSS.
\end{abstract}

\section{Introduction}

Platelets play an essential role in thrombosis and hemostasis. Without nuclei, they are the smallest formed elements of the blood in mammals, and are produced from giant polyploid precursors, the megakaryocytes. ${ }^{1}$ Megakaryocytes differentiate from hematopoietic stem cells in the hematopoietic sites (bone marrow, yolk sac, fetal liver and spleen). It is well accepted that hematopoietic stem cells give rise to megakaryocyte-erythrocyte progenitors in the first instance, and these progenitors commit to both erythroid and megakaryocytic lineage cells. ${ }^{2-4}$ During thrombopoiesis, megakaryocytes then arise from committed megakaryocytic precursors, undergo several cycles of endomitosis to become polyploid and release platelets as cell fragments into the bloodstream. ${ }^{5}$ When a blood vessel ruptures, adherent platelets become activated and initiate the platelet aggregation process, so the circulating platelets adhere to different components of vascular subendothelial structures to stop the bleeding. ${ }^{3}$

Functional platelets require a series of platelet membrane receptors properly expressed, as these receptors are es- sential for platelet adhesion to form clots on the damaged vessel wall and trigger transmembrane signaling leading to cell aggregation and activation. The glycoprotein Ib-IX$V$ complex (GPIb-IX-V), expressed in platelets and megakaryocytes, belongs to the leucine-rich repeat family of membrane proteins. The complex consists of four distinct transmembrane polypeptide subunits, ${ }^{6}$ of which GPIb $\alpha$, GPIb $\beta$, and GPIX are all necessary for efficient biosynthesis of the receptor, whereas GPV is more loosely associated.7,8 When blood vessels rupture causing bleeding, the GPIbIX-V complex mediates platelet attachment to the sites of blood vessel wall injury and activates the platelets. ${ }^{9}$

Bernard-Soulier syndrome (BSS), a rare autosomal recessively inherited bleeding disorder also known as hemorrhagiparous thrombocytic dystrophy, ${ }^{7-9}$ is caused by quantitative or qualitative defects within the membrane GPIb-IX-V complex. ${ }^{6}$ The syndrome is characterized by thrombocytopenia, giant platelets with the absence of platelet aggregation in response to ristocetin, and a range of sometimes life-threatening mucocutaneous bleeding disorders. ${ }^{7,9}$ Mutations in GP1BA (GPIb $\alpha$ ), GP1BB (GPIb $\beta$ ), and GP9 (GPIX) have all been reported in BSS patients, ${ }^{10}$ 
with the reported mutation frequency being higher in GP9 than in the other two genes in a study of 211 BSS families. ${ }^{10}$ GPIX is a subunit of the platelet membrane glycoprotein complex, and multiple point mutations in the GP9 gene are reported to be associated with BSS in patients. ${ }^{10,11} \mathrm{Sev}$ eral GP9 point mutations, such as GP9 70T>C and GP9 $182 A>G$, have been reported to affect protein conformation and consequently attenuate the GPIb-IX-V complex component expression, but there is still a lack of in vivo functional evidence on whether these mutations affect thrombocytes. ${ }^{10,12-15}$ Disruption of GPIX in mice results in thrombocytopenia with giant platelets and prolonged bleeding phenotypes similar to human BSS, while intrauterine embryogenesis makes mice difficult to manipulate during early developmental stages. ${ }^{16}$ Whether and how GP9 participates in the regulation of embryonic thrombocyte development remains incompletely known.

Zebrafish (Danio rerio) have been used as powerful vertebrate models for developmental biology and for modeling many human diseases. Their high fecundity, external development and their optical transparency during early development facilitate high-throughput genetic and small-molecule screening, especially for in vivo phenotype fast-reading in early developmental stages. Importantly, thrombocytes in early vertebrates are equivalent to mammalian platelets in functional and regulatory terms. ${ }^{17,18}$ Thrombocytopoiesis has been described in zebrafish and has demonstrated conservation of regulatory factors and similar developmental processes as those in mammals. ${ }^{19,20}$ Thrombocyte disorders including congenital amegakaryocytic thrombocytopenia, inherited thrombocytopenia, essential thrombocythemia and several platelet functional disorders have been modeled successfully in zebrafish. ${ }^{21}$ ${ }^{25}$ In addition, thrombocytic lineage-specific transgenic reporter strains of zebrafish are available, such as the $T g$ (cd41:eGFP) and $T g$ (mpl:eGFP) lines. ${ }^{19,21}$ Thrombocyte function can be detected by the efficiency of clotting, measured as the time to occlusion or the thrombus surface area, both having been well-established in zebrafish. ${ }^{21,26}$ As multiple mutations in GP9 are associated with clinical manifestations of BSS, understanding the functions and underlying mechanisms of GP9 in thrombocytopoiesis and establishing a proper zebrafish model of BSS would be valuable to understand the disease pathogenesis and to verify the clinical significance of patient-derived GP9 variants.

In the current study, we targeted the gp9 gene using CRISPR-Cas9 technology to generate an inherited BSS zebrafish model. The gp9sMU15 zebrafish modeled BSS well, as it displayed thrombocytopenia from early development to adult stage, thrombocytic progenitor expansion in adult hematopoiesis, and exhibited bleeding disorders. We further demonstrated that human GP9 mirrored the thrombocyte phenotypes in the zebrafish model, and pa- tient-derived GP9 ${ }^{70 T>C}$ and GP9 ${ }^{182 A>G}$ mutations are thrombocytopoietic deficient mutations responsible for human BSS. Moreover, we found that recombinant human thrombopoietin (rhTPO), used clinically for promoting thrombocytopoiesis, was also effective for relapsing BSS thrombocytopenia phenotypes in the model. Interestingly, we found that decitabine, used for treating leukemia and idiopathic thrombocytopenia purpura in the clinic, could also effectively alleviate thrombocytopenia in BSS zebrafish. Thus, gp $9^{\text {SMu15 }}$ zebrafish could serve as an ideal model for understanding the deficiency of thrombocytopoiesis and hemostasis associated with BSS, and it could be applied as a useful tool for fast clarifying human patient-derived GP9 variants of unknown clinical significance, as well as for the development of therapeutic strategies for BSS.

\section{Methods}

\section{Generation of gp $9^{\text {SMU15 }}$ mutant lines}

We generated gp9sMu15 mutants using the CRISPR/Cas9 method. The zebrafish gp9 target within exon 2 is 5'GGGCAAAGTCACGCACCTGC-3'. gp9 guide RNA was transcribed in vitro by using mMESSAGE MMACHINE kit (Ambion). We generated gp9 mutants by injecting one-cell stage embryos with Cas9 protein (EnGen Cas9 NLS, NEB) and gRNA. FO fish were crossed with wild-type (WT) fish to produce F1 progeny, which were then genotyped and sequenced to identify the inheritable mutation. We acquired the main frameshift mutations with the deletion of 17 bp (F1). We crossed the heterozygotes F1 gp ${ }^{\text {sMu15/+ }}$ to generate homozygous mutant F2 gp9smu15. All work involving zebrafish was reviewed and approved by the Animal Ethics Committee or the Animal Research Advisory Committee of Southern Medical University and South China University of Technology

\section{Analysis of thrombocyte function}

We monitored the time to stop bleeding or the bleeding area size in adult zebrafish and the time to occlusion in zebrafish larvae after injury. Comparable cuts were made in WT siblings and gp9smu15 mutants with a scalpel blade at the junction region between the torso and the tail fin to destroy the vessels near the tail part; the cuts were performed in a single-blind manner to exclude the subjective factor of operation, and the fish were genotyped after the injury. The time to stop bleeding (the time the injury was made to the time the bleeding stopped) was measured under a microscope and recorded by a video camera. Sodium hydroxide $(\mathrm{NaOH})$ treatment to induce gill bleeding was performed as previously described with some modifications. ${ }^{27,28}$ We placed zebrafish in a glass dish containing $15 \mathrm{~mL}$ of $20 \mathrm{mM} \mathrm{NaOH}$ solution, and the process was recorded under a microscope (Carl Zeiss Meditec AG, Jena, 
Germany). $\mathrm{FeCl}_{3}$ treatment was performed as previously described with some modifications. ${ }^{26,29}$ In detail, 6-day post-fertilization (dpf) larvae, with various genotypes Tg(cd41:eGFP);WT siblings and Tg(cd41:eGFP);gp 9sMU15 were immobilized in $0.8 \%$ low-melt agarose gel and $0.02 \%$ anesthetic tricaine (Sigma-Aldrich). A drop (2.5 $\mu \mathrm{L}$ ) of $1 \% \mathrm{FeCl}_{3}$ was placed on the tail region. From the time the first drop appeared on the tail region to the accumulation of the

A

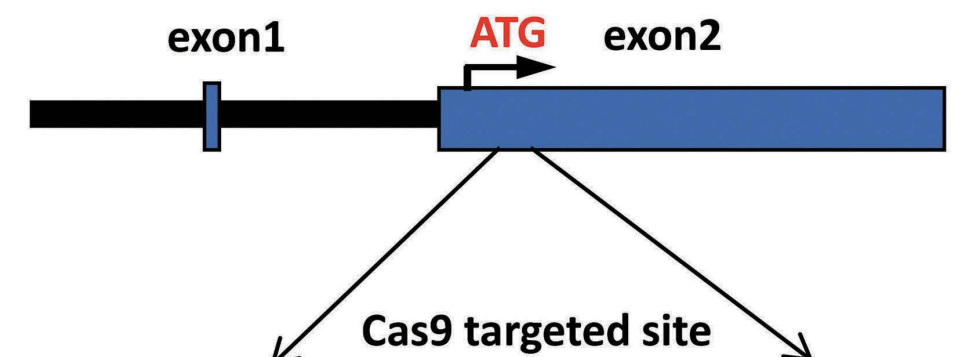

B
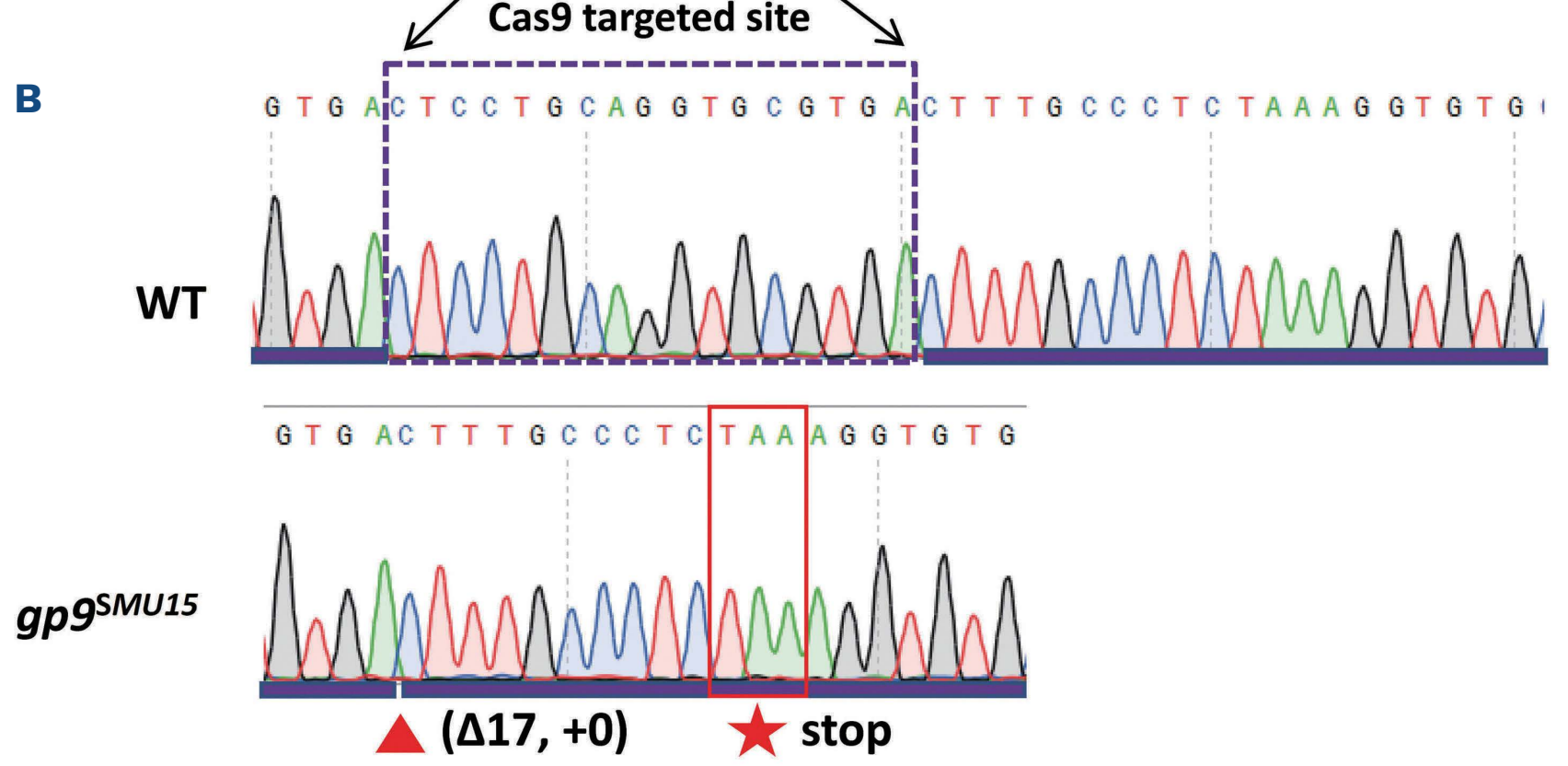

C

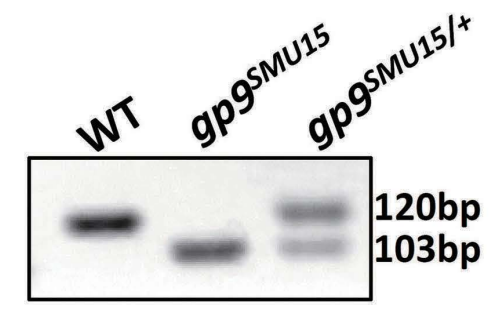

D

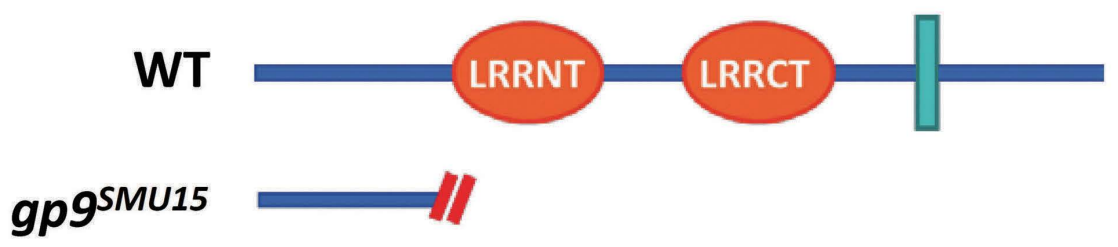

Relative mRNA level

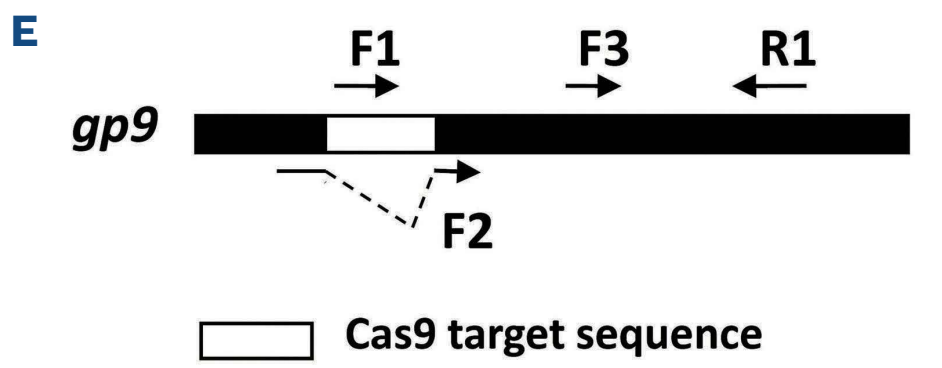

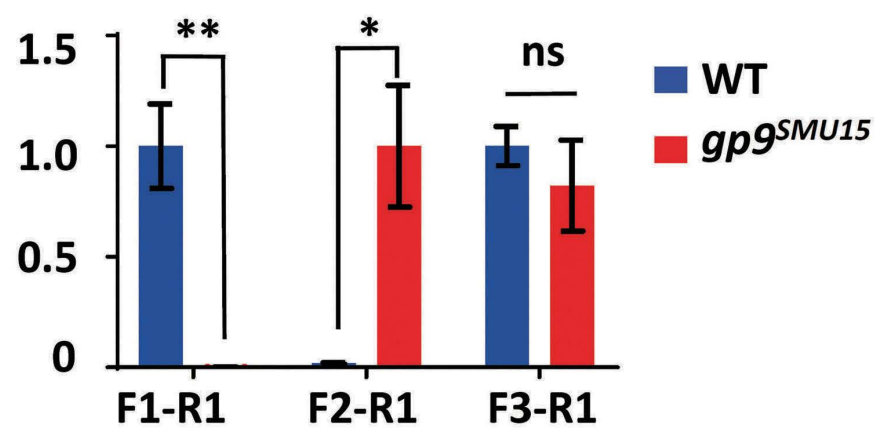

Figure 1. Generation and characterization of gp9 9Mu15 $^{\text {zebrafish }}$ using CRISPR/Cas9. (A) The zebrafish gp9 gene structure. The start coding region is shown with ATG. The targeting sequence aimed at the coding region is shown in the purple dashed box. (B) Sanger sequencing identified the wild-type (WT) sequence (top) and 17-bp deletion (red arrowhead) in gp9smu15. Frameshift mutation of gp 9 creates premature stop codons indicated with the red asterisk. (C) Agarose gel pictures of the polymerase chain reaction product from WT, mutant, and heterozygote zebrafish. Lane 1: the wild type bands; lane 2: the mutant bands; lanes 3: the heterozygote bands. (D) Gp9 protein structures in WT and mutant fish. The Gp9 protein analyzed with the SMART program contains one leucine-rich repeat N-terminal (LRRNT) domain, one leucine-rich repeat C-terminal (LRRCT) domain and one transmembrane domain. Orange ovals indicate a LRRNT or LRRCT domain, the green box indicates the transmembrane domain. Red slashes indicate the premature stop of Gp9 protein. (E) gp9 ${ }^{17,+0}$ mutated transcripts generated in gp9smu15 mutants. Primer pairs F1 (wt_FP) F2 (mut_FP) F3 (common_FP) and R1 (common_RP) were utilized for detecting the WT form and gp9 17,+0 form, respectively. Statistical significance was determined using a two-sample Student $t$-test, $n \geq 10$ per group, data were combined from four biological replicates, mean \pm standard error of mean. ${ }^{*} * P<0.01$; ${ }^{*} P<0.05$; ns: not significant. 
thrombocytes at the injury site, the time to occlusion was measured at the microscopic stage monitored by a fluorescence microscope.

\section{Drug treatment}

Tg(cd41:eGFP) sibling and Tg(cd41:eGFP);gp 9sMu15 transgenic mutant zebrafish were utilized. Embryos were soaked into egg water containing drugs in appropriate concentration at 36 hours post-fertilization (hpf) using 12-well plates and scored at $4 \mathrm{dpf}$ by counting the cd41:eGFPhigh-labeled thrombocytes to determine whether drugs were effective. Fifteen embryos were put in each well with a volume of 3
A

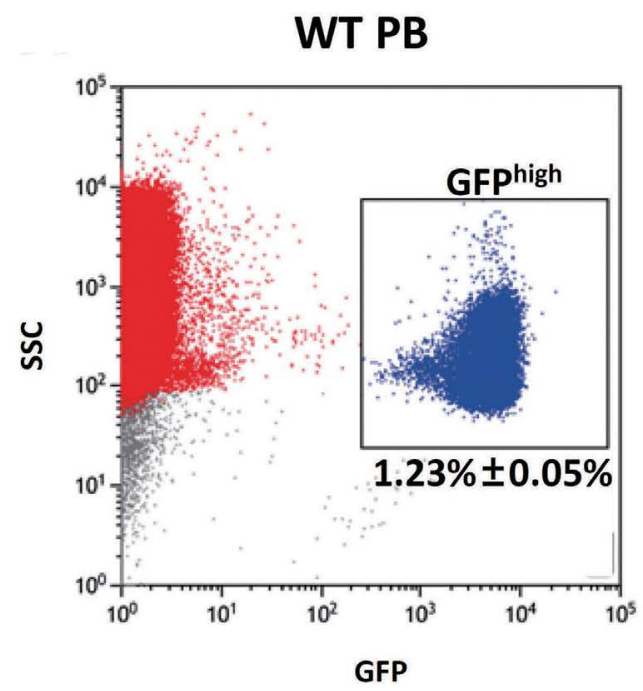

C

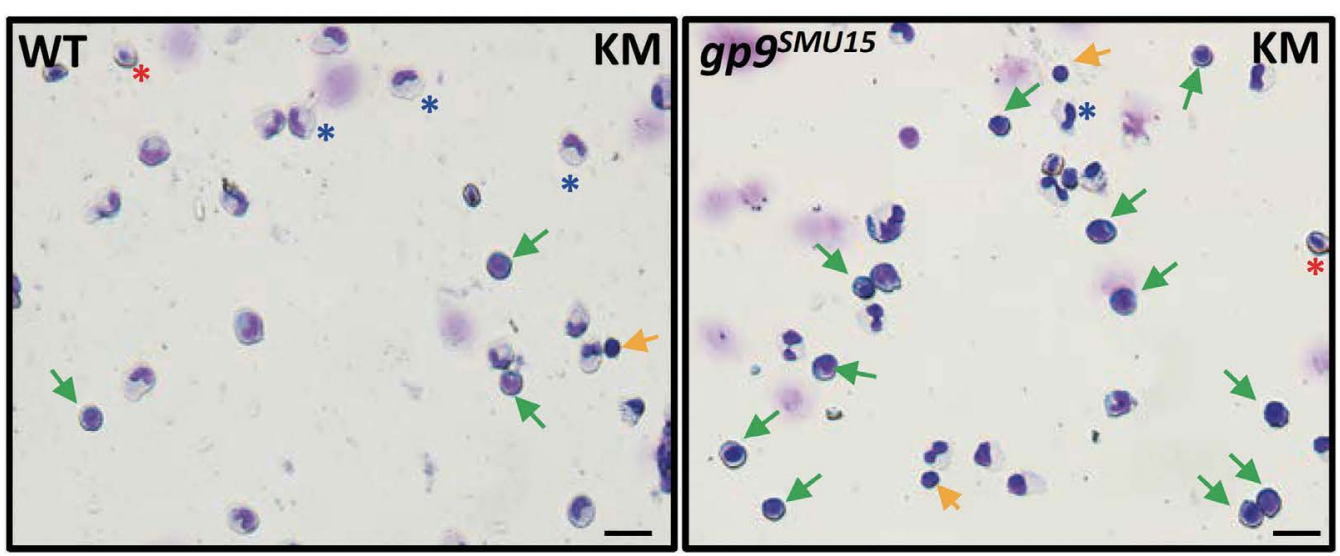

$\mathbf{E}$

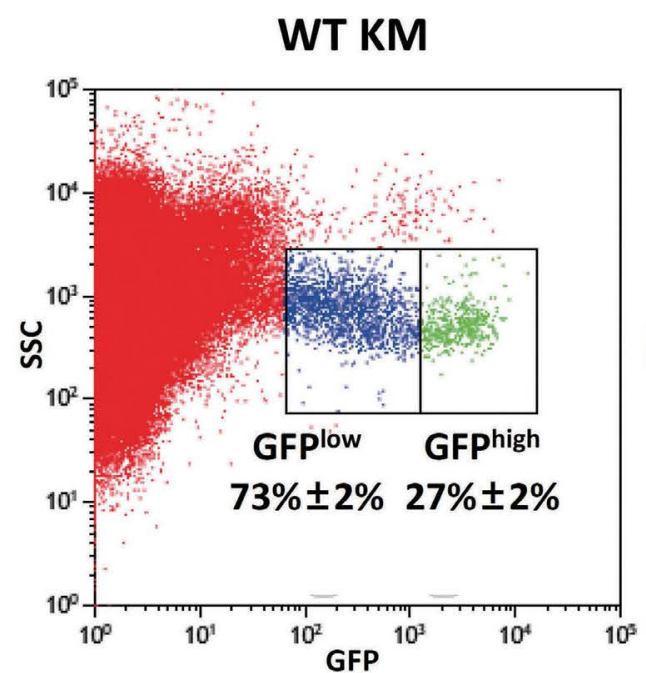

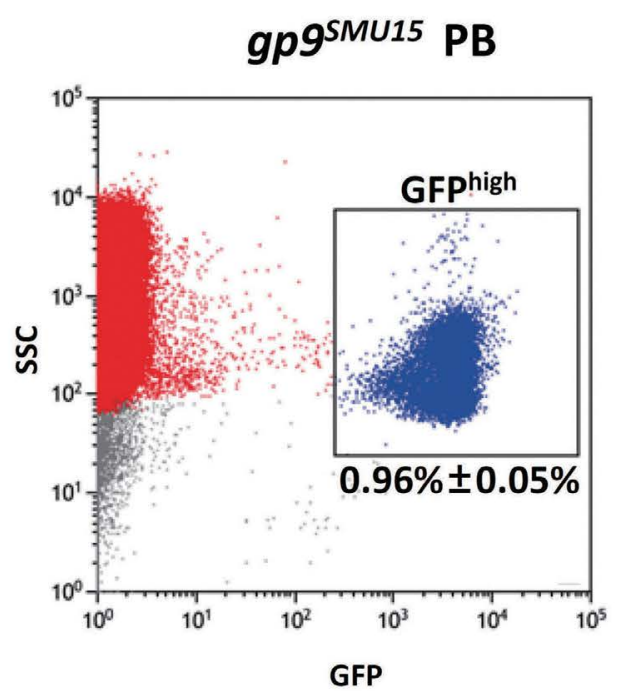

B

\section{cd41:eGFPhigh cell percentage in PB (adult)}

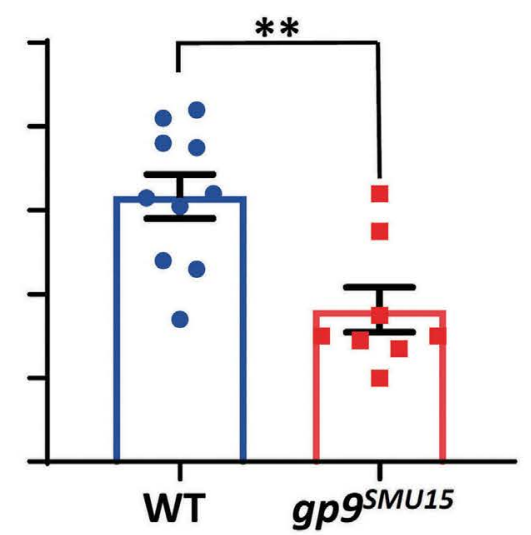

D

\section{Cell percentages in KM}

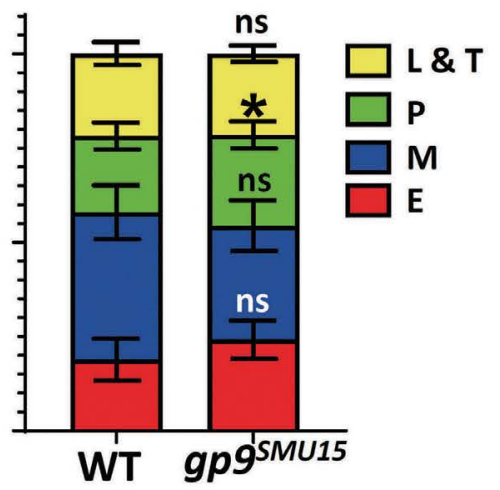

F

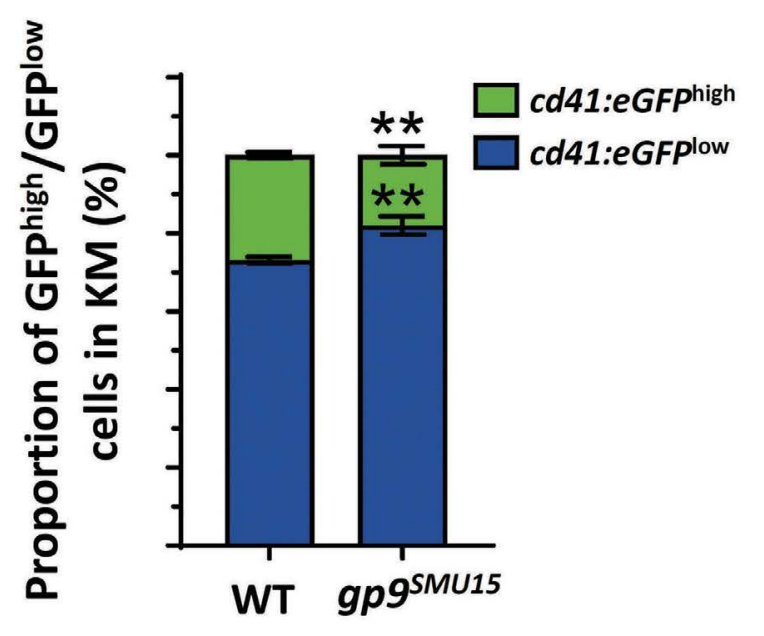

Figure 2. gp $9^{\text {sMu15 }}$ adult fish display thrombocytopenia and abnormal precursor expansion. (A) Flow cytometry analysis of peripheral blood (PB) cells in wild-type (WT) and gp $9^{\text {SMU15 }}$ fish. FITC was directly proportional to 488-GFP cells and side scatter (SSC) was indicative of cellular granularity. (B) Percentage of cd41:eGFP+ thrombocytes in PB determined by flow cytometry (Student $t$-test, $\mathrm{n}=6$; mean \pm standard error of mean [SEM]; ns: not significant; ${ }^{*} P<0.05$ ). (C) May-Grünwald-Giemsa staining of kidney marrow (KM) cells in WT and gp9smu15 fish. The scale bar represents $20 \mu \mathrm{m}$. Arrowheads are colored yellow for lymphocytes or thrombocytes and green for precursors. Asterisks are colored blue for myelomonocytes and red for erythrocytes. (D) Blood cell counts of WT and gp9 ${ }^{\text {sMu15 }} \mathrm{KM}$ by May-Grünwald-Giemsa staining. L\&T: lymphocytes and thrombocytes; P: precursors; M: myelomonocytes; E: erythrocytes. (Student $t$-test, $n=6$; mean \pm SEM; ns: not significant; ${ }^{*} P<0.05$ ). (E) Flow cytometry analysis of KM cells in WT and gp9 $9^{\text {sMU15 }}$ fish. FITC was directly proportional to 488-GFP cells and SSC was indicative of cellular granularity. (F) Percentage of cd41:eGFPlow and cd41:eGFPhigh thrombocytes in KM cells determined by flow cytometry (Student $t$-test, $\mathrm{n}=6$; mean $\pm \mathrm{SEM}$; ns: not significant; $* \star P<0.01$ ). 
$\mathrm{mL}$ solution. To obtain a proper working concentration of rhTPO (rhTPO injection; 3SBIOINC, Shenyang, China) and decitabine (Selleck S1200; Selleck, Houston, TX, USA), we treated 1.5-dpf WT embryos with gradient concentrations of rhTPO $(50,100$ and $500 \mathrm{U} / \mathrm{mL})$ or decitabine $(10,20,50$ and $100 \mu \mathrm{M}$ ) until $4 \mathrm{dpf}$ to see whether zebrafish thrombocytes could respond to the concentrations. Doses of 100 $\mathrm{U} / \mathrm{mL}$ rhTPO and $20 \mu \mathrm{M}$ of decitabine were chosen as the concentrations that could elevate WT thrombocytes efficiently but not cause developmental retardation in larvae.

\section{Results}

\section{The gp $9^{\text {SMU15 }}$ adult fish display thrombocytopenia and hematopoietic precursor expansion}

To investigate the role of gp9 in thrombocytopoiesis, we generated a gp9-deficient zebrafish mutant by targeting exon 2 of the gp9 gene using CRISPR-Cas9 technology (Figure $1 \mathrm{~A})$. We obtained an FO mutant line containing gp9 (-17 bp, +0) (Figure 1B, C), and outcrossed this line with WT zebrafish to generate $\mathrm{F} 1$ heterozygous and F2 homozygous progenies. The gp9 $(-17 \mathrm{bp},+0)$ mutant was predicted to encode a truncated Gp9 protein lacking the two-leucine rich repeat domains and the transmembrane domain (Figure 1D). Using quantitative real-time polymerase chain reaction to detect gp9 mRNA, we found that the mutant fish produced only the mutated mRNA (Figure 1E). Therefore, gp9 (-17 bp, +0) (gp $9^{\text {SMU15 }}$ hereafter) was predicted to be a loss-of-function mutant.

To characterize the effects of gp9 disruption on thrombocytopoiesis in zebrafish, we determined thrombocyte numbers in gp $9^{\text {sMu15 }}$ mutants and their siblings by monitoring cd41:eGFP expression in the $T g(c d 41: e G F P)$ line, as the vast majority of circulating thrombocytes could be recognized by flow cytometry analysis (FACS) with bright cd41:GFPhigh fluorescence in adult fish. ${ }^{19}$ Notably, although gp $9^{\text {sMu15 }}$ mutants were viable and able to survive to adulthood, the gp9smu15 adult mutants displayed thrombocytopenia as the number of circulating cd41:eGFPhigh thrombocytes in adult mutants was about $78 \%$ of the number in adult WT sibling fish (Figure 2A, B). These data indicate that gp9sMu15 zebrafish displayed thrombocytopenia in the adult stage, resembling BSS in human patients. To directly examine BSS-like hematologic disorders in gp9 $9^{\text {sMu15 }}$ adult fish, kidney-marrow (KM) cells were collected from gp9smu15 adult fish or siblings and subjected to cytological analyses and blood cell count. We found a significant expansion of the precursor cell population in adult gp9 $9^{\text {SMU15 }}$ fish (Figure 2C, D). Recent single-cell RNA-sequencing of zebrafish adult KM revealed the continuous nature of thrombocyte development, ${ }^{30}$ and FACS could recognize hematopoietic stem and progenitor cells (HSPC) to differentiated thrombocytes with weak to bright cd41:eGFP
(GFPlow to GFPhigh) fluorescence in the adult KM cells. ${ }^{19,31,32}$ To compare the thrombocyte population of gp $9^{\text {sMU15 }}$ fish with that of their WT siblings in KM, cd41:eGFP ${ }^{+}$cells were isolated by FACS. In accordance with the cytological results (Figure 2C, D), the FACS results also showed an increase in the cd41:eGFPlow cell population from $73 \%$ in the adult WT sibling fish to $82 \%$ in adult gp $9^{\text {SMu15 }}$ fish (Figure $2 \mathrm{E}, \mathrm{F}$ ), suggesting that gp $9^{\text {sMu15 }}$ fish had a higher percentage of cd41:eGFPlow HSPC than had their WT siblings. The morphological analysis revealed that there were no obvious cell size changes within the cd41:eGFPlow HSPC population (Online Supplementary Figure S1A, C). However, the populations of cd41:eGFPhigh thrombocytes in gp9sMu15 mutant KM and peripheral blood were both larger than those in their WT siblings (medium vs. small) (Online Supplementary Figure S1A-D). The above data demonstrate that gp9sMu15 zebrafish mutants probably have a developmental block, with expansion of larger thrombocytes and reduced small-nucleated thrombocytes in adulthood. BSS patients display increased bone marrow megakaryocytes but reduced mature platelets. ${ }^{34}$ In our study, zebrafish gp9 $9^{\text {smu15 }}$ mutants showed a similar expansion of the progenitor population but reduced circulating thrombocytes.

\section{The gp9 $9^{S M U 15}$ mutants display impaired thrombocyte function}

BSS patients generally have moderate thrombocytopenia, giant platelets and a bleeding tendency. They may suffer severe hemorrhage following injury or during surgery. ${ }^{33}$ Under normal conditions, WT zebrafish rarely bleed severely, whereas $\sim 21 \%$ (4/19) of gp $9^{\text {sMu15 }}$ mutants had blood spots on the body or near the tail fins (Figure $3 \mathrm{~A}$ ), indicating that the gp9 mutation led to a pronounced bleeding tendency. Thrombocyte function can be assessed by the ability to stop bleeding and to clot, which has been wellestablished in the zebrafish model. ${ }^{21,29}$ By calculating the time to stop bleeding after a tail-cut injury, we found that the average time in gp $9^{\text {smu15 }}$ adult mutants was $\sim 50 \mathrm{~s}$, much longer than in the WT group ( 20 s) (Figure 3B, C). We also used $\mathrm{NaOH}$ as a substance to create chemical damage to induce gill bleeding. ${ }^{27,28}$ We found more severe gill bleeding in gp $9^{\text {SMU15 }}$ fish than in WT fish (Figure 3D, E). We then monitored the accumulation of cd41:eGFPhigh thrombocytes following administration of $\mathrm{FeCl}_{3}$ to induce an oxidative injury to the vascular endothelium, ${ }^{26,29}$ and found that the time to accumulation of thrombocytes at the injured venule in gp9 ${ }^{\text {sMu15 }}$ zebrafish larvae was significantly longer (mean \pm standard error of mean [SEM], 36.4 $\pm 5.2 \mathrm{~s}$ ) than in the WT sibling controls (mean \pm SEM, 22.2 $\pm 2.4 \mathrm{~s}$ ) (Figure $3 \mathrm{~F}$ ). Taken together, these results demonstrated that the thrombocytopenic gp9 $9^{\text {SMU15 }}$ zebrafish exhibited bleeding disorders similar to the extended clinical manifestations of clotting and prolonged bleeding time in BSS patients. 
A
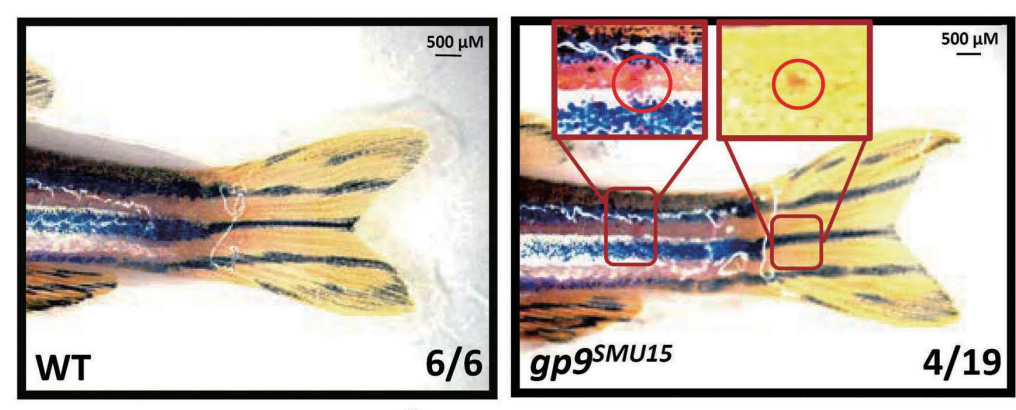

B
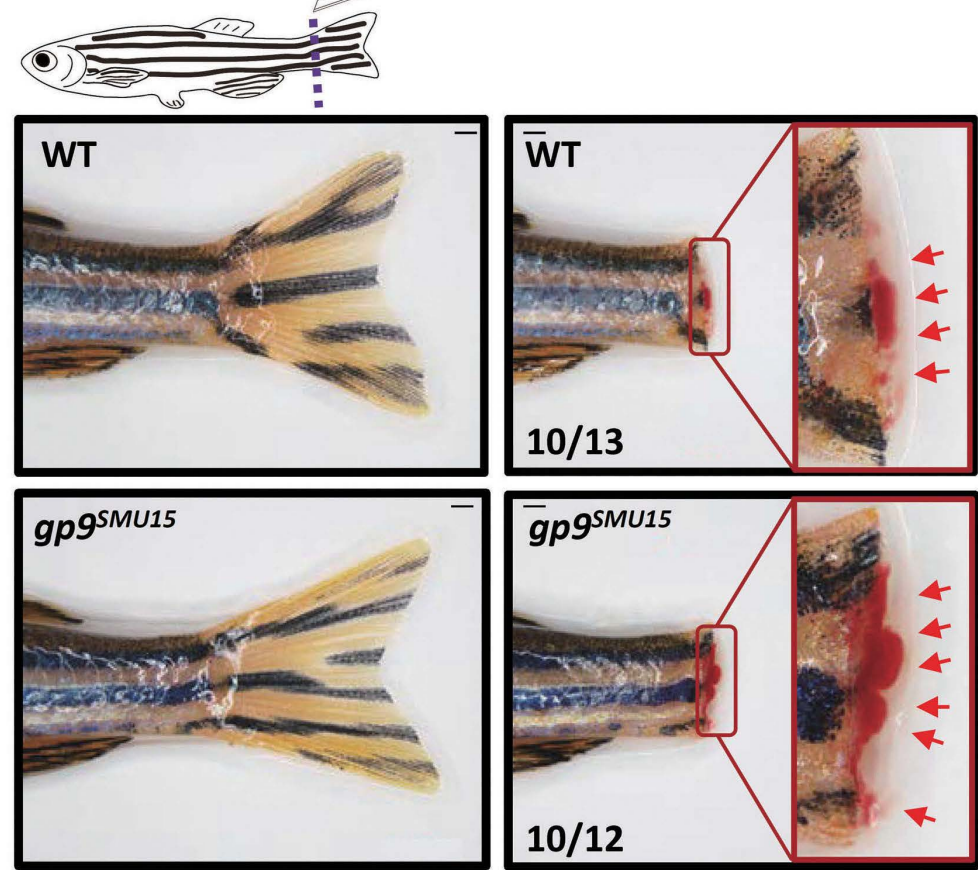

D
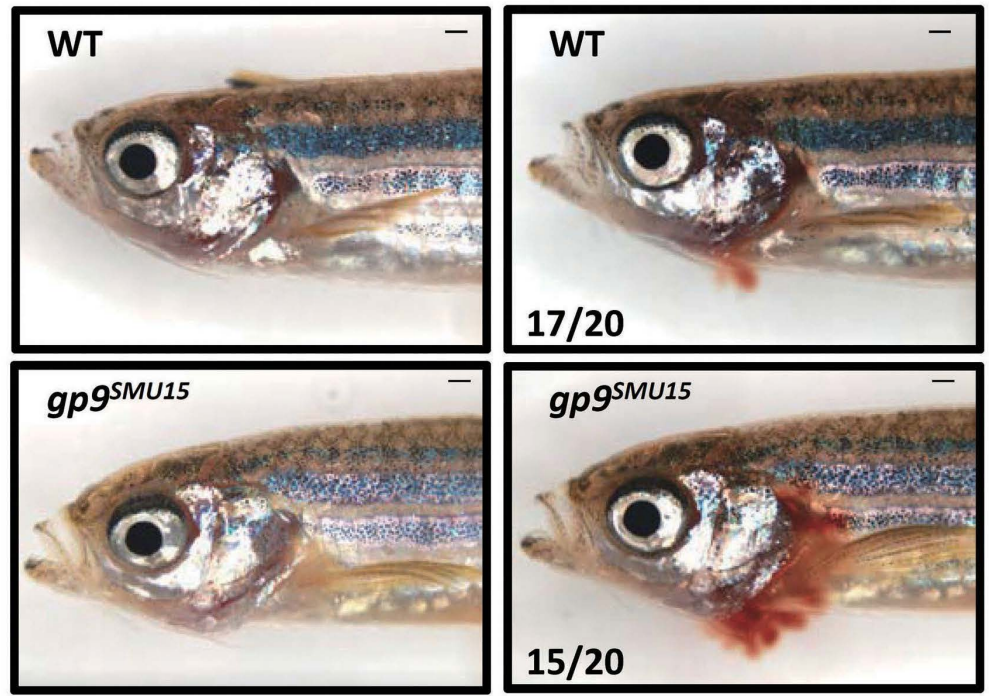

C Time to stop bleeding (seconds)

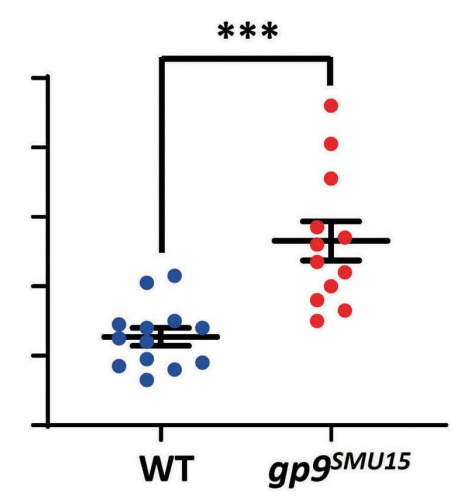

E

The numbers of pixels

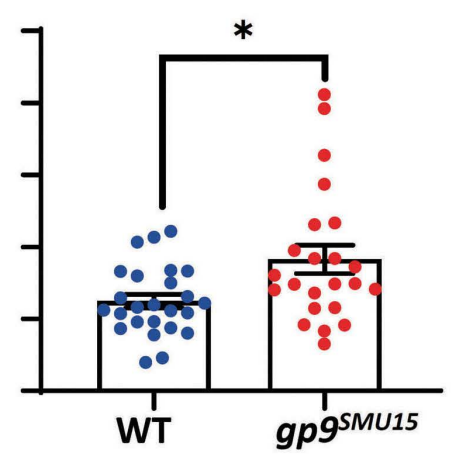

$\mathbf{F}$
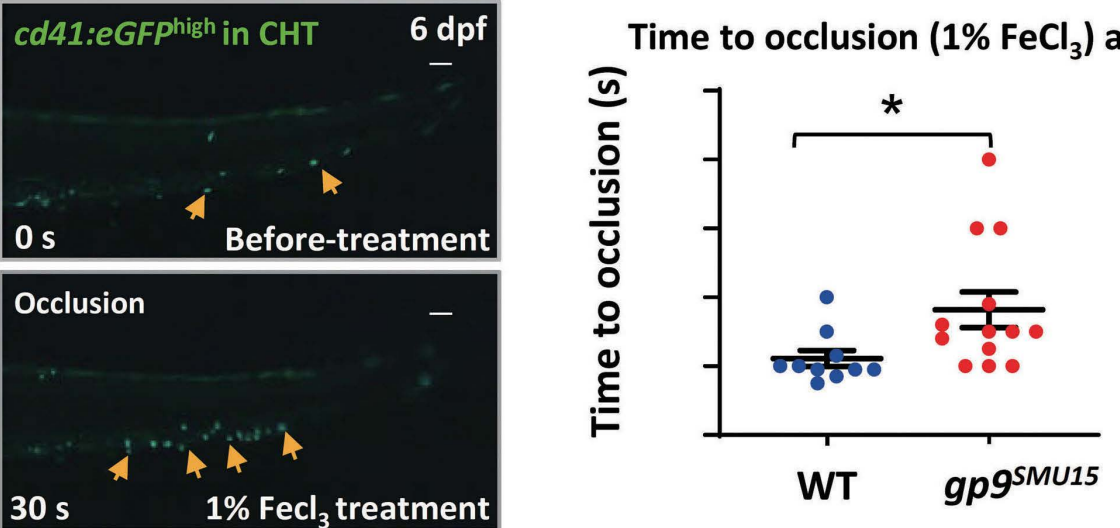

Figure 3. gp9 $9^{\text {smu15 }}$ mutants display impaired thrombocyte function. (A-D) Clotting in adult wild-type (WT) siblings and gp9smu15 mutants. Representative images, under normal conditions, of blood spots on the zebrafish body or near the tail fins (A). The time to stop bleeding in adult WT siblings (upper) and gp9smu15 mutants (lower) (B). (C) Quantitative data showing the time to stop bleeding in WT and gp9smu15 siblings (D) Representative images of gill bleeding after injury in the sibling and gp9smu15 mutants. The representative pictures were captured at $90 \mathrm{~s}$. (E) Quantitative data showing the red pixels around the gill in WT and gp 9 smu15 fish The red color pixels, indicating the extent of bleeding, were counted by Adobe photoshop CC software. (F) Zebrafish after injury induced by $1 \%$ ferric chloride: quantitative data showing the time to accumulation of thrombocytes at the injured venule. Statistical significance was determined by a two-sample Student $t$-test, $n \geq 10$, mean \pm standard error of mean, $\star P<0.05$. Scale bars: $500 \mu \mathrm{m}$. 
The gp9-disruption led to thrombocytopenia in zebrafish larvae

To characterize the effects of gp9 disruption on thrombocytopoiesis in zebrafish embryos or larvae, we determined thrombocyte numbers in gp $9^{\text {smu15 }}$ mutants and their siblings by monitoring cd41 mRNA and cd41:eGFP expression. In zebrafish larvae, two distinct populations of cd41:eGFP cells could be recognized, cd41:eGFPlow precursor and progenitor cells and the cd41:eGFPhigh thrombocytes, by FACS or by whole mount observation under a fluorescence microscope. ${ }^{19,32,34}$ We isolated $\mathrm{GFP}^{+}$cells from 3-dpf embryos by FACS, and found that the cd41:eGFPhigh cell population was significantly decreased but that the cd41:eGFPlow cell population was increased in 3-dpf gp $9^{\text {sMu15 }}$ embryos (Figure 4A, B), suggesting that thrombocyte differentiation was affected by the gp9 mutation. Furthermore, quantification analysis showed that, at $4 d p f$, signals of cd41:eGF$P^{\text {high }}$ cells in the caudal hematopoietic tissue $(\mathrm{CHT})$ region were markedly reduced in gp9smu15 mutant larvae compared with the signals from WT sibling embryos (Figure 4C, D), indicating that gp9smu15 mutants exhibited thrombocytopenia during embryonic development. This conclusion was further supported by the downregulation of several thrombocyte-related genes, such as cd41 and genes required for thrombocytopoiesis (fog1 and $n f e 2$ ) (Figure 4E). Notably, the aorta-gonad-mesonephros (AGM) cd41:eGFPlow cells, mainly represented by HSPC, were unaltered in 2-dpf gp $9^{\text {sMu15 }}$ mutants (Figure 4F), and cmyb expression was unaltered in the AGM and $\mathrm{CHT}$ regions (Figure $4 G$ ), suggesting that HSPC were unaffected by gp9 mutations. Other definitive blood lineages were also unaffected, as indicated by unaltered $\mathrm{mpo}^{+}$neutrophils at 3-dpf, ße1globin ${ }^{+}$and $\mathrm{O}$-dianisdine ${ }^{+}$erythrocytes, and rag $1^{+}$lymphocytes at 5-dpf (Online Supplementary Figure S2A-E). Furthermore, primitive blood lineages including embryonic erythroid markers (ae1-globin and gata1) and a primitive myeloid marker (pu.1) were unaffected in gp $9^{\text {SMU15 }}$ mutants (Online Supplementary Figure S3A-D). Taken together, these results show that gp $9^{\text {SMu15 }}$ mutants exhibit a lineage-specific thrombocytic deficiency without other blood lineage cells being affected.

To rule out the possibility of off-target mutations, we overexpressed Gp9-eGFP fusion protein under the control of the heat-shock promoter ( $h s p: g p 9$-egfp) and showed that heat-shock-induced gp9 overexpression could protect gp9smu15 mutants from thrombocytopenia (Online Supplementary Figure $S 4 A-C$ ). These data demonstrate that the loss of gp9 function leads to thrombocytopenia in zebrafish larvae.

\section{Thrombocytopenia in gp9 $9^{\text {SMU15 }}$ mutants is caused by reduced proliferation of thrombocyte precursors}

To explain the cellular basis of the thrombocytopenia in gp $9^{\text {sMu15 }}$ mutants, we further explored whether thrombo- cyte proliferation is reduced or apoptosis increased in gp9-deficient mutants. Thus we monitored the $\mathrm{CHT}$ region cd41:eGFP positive cell growth or death changes in the

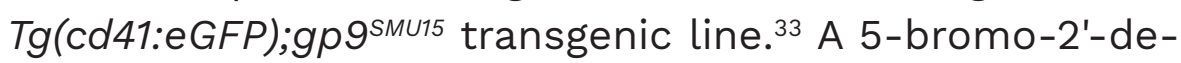
oxyuridine (BrdU) pulse labeling incorporation assay revealed that the percentage of BrdU-positive cd41:eGFP+ cells in total cd41:eGFP+ cells in the CHT was markedly reduced in gp9smu15 mutants at $3 \mathrm{dpf}$, and the decreased population were BrdU-labeled cd41:GFPlow cells rather than cd41:GFPhigh cells, suggesting that the proliferation of cd41:eGFPlow hematopoietic precursors or thrombocyte progenitor cells was affected by the gp9 mutation (Figure $5 \mathrm{~A}, \mathrm{~B})$. To investigate the apoptosis of thrombocytes in gp $9^{\text {sMu15 }}$ mutants, we performed a terminal deoxynucleotidyl transferase dUTP nick-end labeling (TUNEL) assay, and found almost no cd41:eGFP/TUNEL double-positive cells in the CHT at $3 \mathrm{dpf}$ in either WT siblings or gp9sMu15 mutants (Figure $5 \mathrm{C}$ ), indicating that thrombocytopenia was not likely caused by apoptosis. These results demonstrated that thrombocytopenia in gp9smu15 mutants could be mainly attributed to reduced proliferation.

\section{Validation of the zebrafish model using human Bernard- Soulier syndrome-related GP9 mutations}

The phylogenetic tree of Gp9 suggests the evolutional conservation from zebrafish to mammals (Online Supplementary Figure S5A). Multiple sequence alignment demonstrates the similarity of GPIX between zebrafish and mammals (Online Supplementary Figure S5B), especially in the ectodomain (with $~ 37 \%$ identity). ${ }^{35}$

Human BSS is reported to be associated with several GP9 mutations, such as GP9 c.70T>C (C24R) and GP9 c.182A>G (N61S) (Online Supplementary Figure S5B), which have been noted to change the GPIX conformation and mildly impair the protein expression of itself and the GPIB-IX-V complex at the molecular level..$^{10,12,13}$ However, there is still a lack of functional evidence for the two mutations affecting thrombocytopoiesis in animal models. To verify the mutation's effects in models, we first studied gp9smu15 zebrafish to see whether human GP9 (hsGP9'TT hereafter) could rescue zebrafish BSS. We found that thrombocytopenia of zebrafish gp9sMu15 mutants could be recovered by overexpression of hsGPgwt (Figure 6A-C), as cd41:eGFPhigh thrombocyte counts in gp9 $9^{\text {smu15 }}$ zebrafish injected with hsGP9 ${ }^{W T}$ mRNA (34.2 \pm 3.2 thrombocytes) increased to almost the normal level in WT siblings (34.6 \pm 1.9 thrombocytes) (Figure 6B, C). These results further confirmed the functional conservation of thrombocytopoiesis in zebrafish and human GP9, and suggested that gp9 ${ }^{\text {SMU15 }}$ zebrafish could be utilized as a BSS model for fast-validating the functional significance of $h s G P 9$ variants. We further overexpressed the human BSS isoform GP9c.70T>C and c.182A>G mRNA (hereafter $h s^{\text {GP970T>C }}$ and $h s^{\text {GP9182A>G, }}$, respectively, as shown in Online Supplementary Figure S5A) 
A

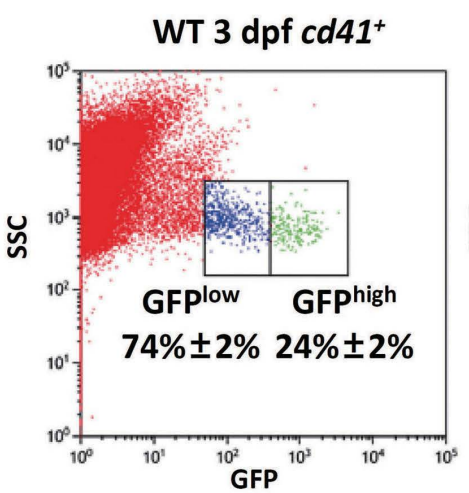

C

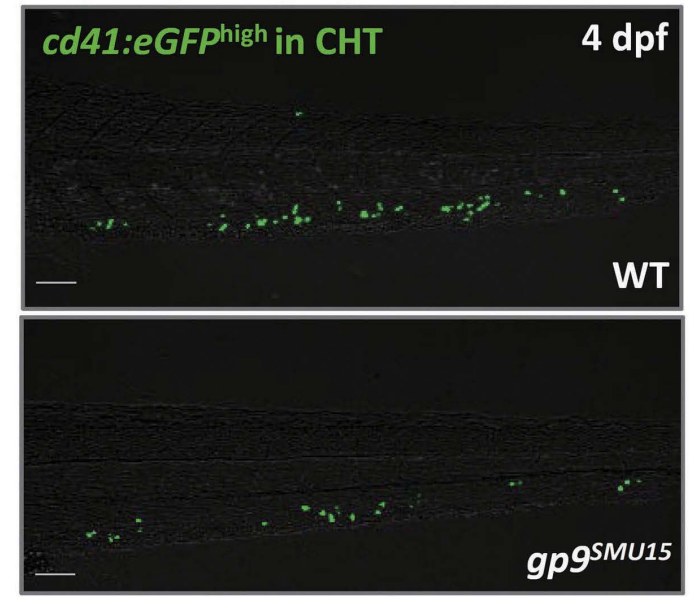

E

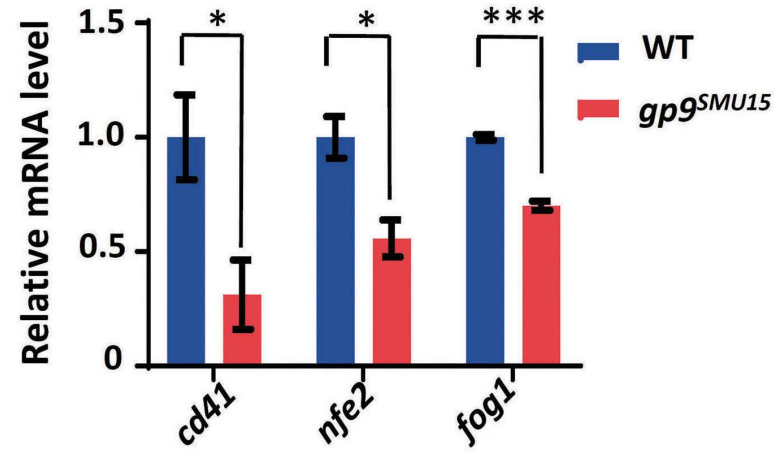

B

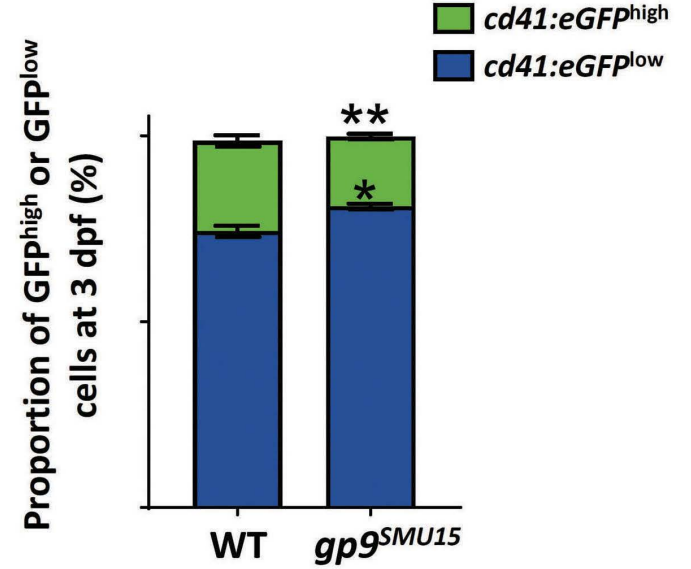

D

No. of 4 dpf cd41:eGFPhigh in CHT

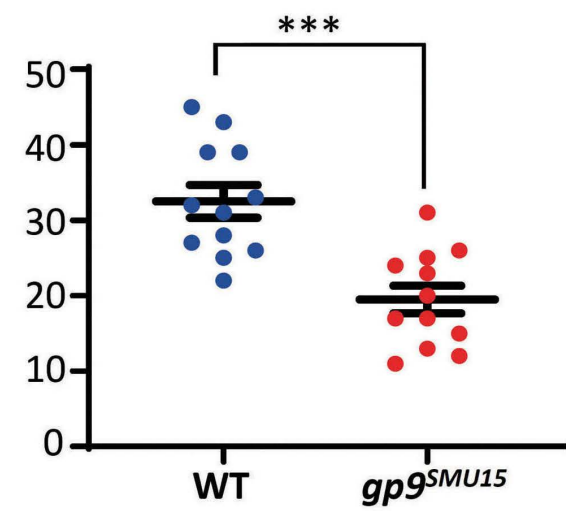

No. of 2 dpf cd41:eGFPlow HSPCs in AGM

$\mathbf{F}$

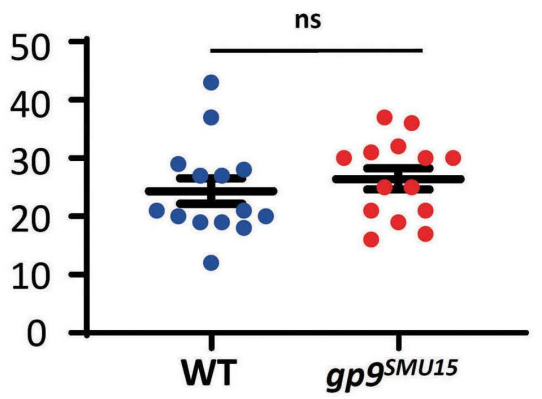

G

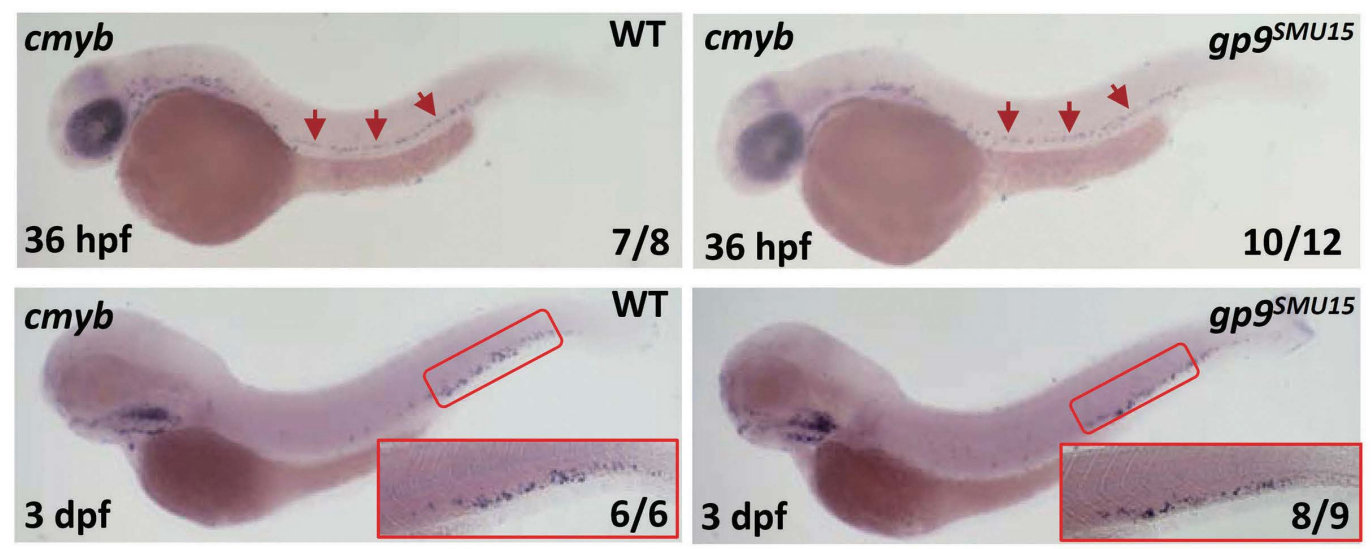

Figure 4. Thrombocytopenia in gp9smu15 zebrafish embryos. (A-D) cd41:eGFPhigh thrombocytes were decreased in gp9smu15 embryos. Flow cytometry analysis of cd41:eGFP ${ }^{+}$cells in 3-day post fertilization (dpf) wild-type (WT) and gp9 ${ }^{\text {SMU15 }}$ embryos. GFP area was directly proportional to 488-GFP cells and side scatter (SSC) was indicative of cellular granularity (A). Percentage of cd41:eGFPlow and cd41:eGFPhigh thrombocytes in 3-dpf embryo cells determined by flow cytometry (Student $t$-test, n>100 embryos per group, and data were combined from three biological replicates, mean \pm standard error of mean [SEM], ns: not significant, $* * P<0.01)$ (B). Representative images for staining of cd41:eGFP protein in $4 \mathrm{dpf} T g\left(\mathrm{~cd} 41\right.$ :eGFP); ${ }^{\mathrm{WT}}$ and mutant Tg(cd41:eGFP);gp9smu15 embryos. Images showed GFPhigh signals (C). Statistical significance was determined using a two-sample Student $t$-test, $\mathrm{n}>10$, mean $\pm \mathrm{SEM}$, $* * * P<0.001$ (D). (E) Relative expressions of thrombocytic markers (cd41, $n f$ f and fog1) in 3dpf WT siblings (blue column) and gp $9^{\text {SMU15 }}$ mutants (red column) by quantitative real-time polymerase chain reaction. Statistical significance was determined using a two-sample Student $t$-test; $n \geq 10$ per group, and data were combined from three biological replicates, mean $\pm \mathrm{SEM}, * P<0.05, * * * P<0.001$. (F) Hematopoietic stem and progenitor cells (HSPC) were not affected in gp9smu15 mutants. Quantification of the aorta-gonad-mesonephros (AGM)-localized cd41:eGFPlow HSPC in gp9smu15 mutants and WT embryos at 2 dpf. Statistical significance was determined using a two-sample Student $t$-test, $n>10$, mean \pm SEM, ns: not significant. (G) Whole-mount in situ hybridization (WISH) of cmyb expression in control (left panel) and gp9smu15 (right panel) embryos at 36 hours post fertilization (hpf) and $3 \mathrm{dpf}$. 
A

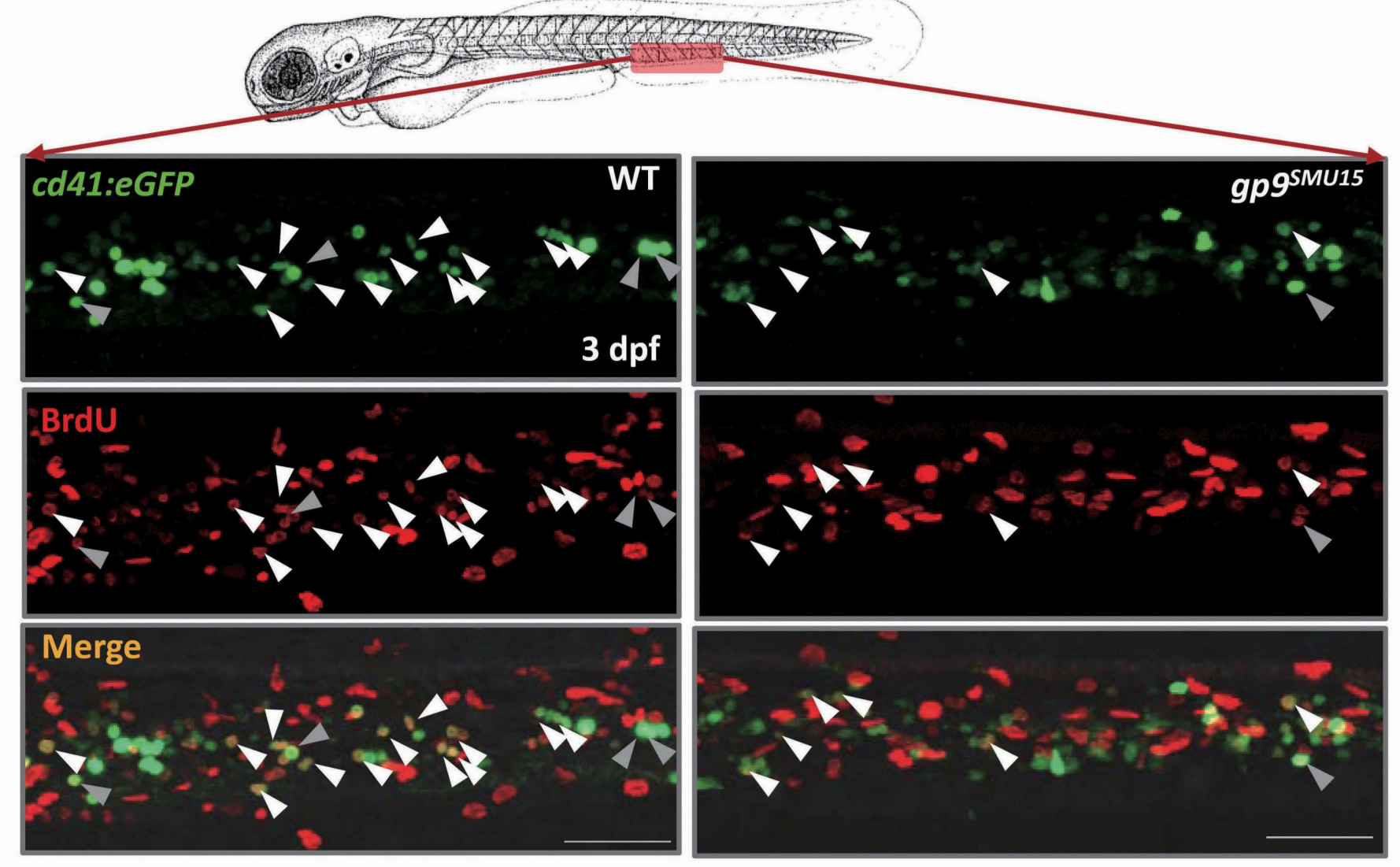

B

cd41 ${ }^{+} \mathrm{BrdU}^{+} / \mathrm{cd} 41^{+}$cell

cd41 ${ }^{\text {high }} \mathrm{BrdU}^{+} /$cd41 $1^{\text {high }}$

cd41 ${ }^{\text {low }} \mathrm{BrdU}^{+} / \mathrm{cd} 41^{\text {low }}$
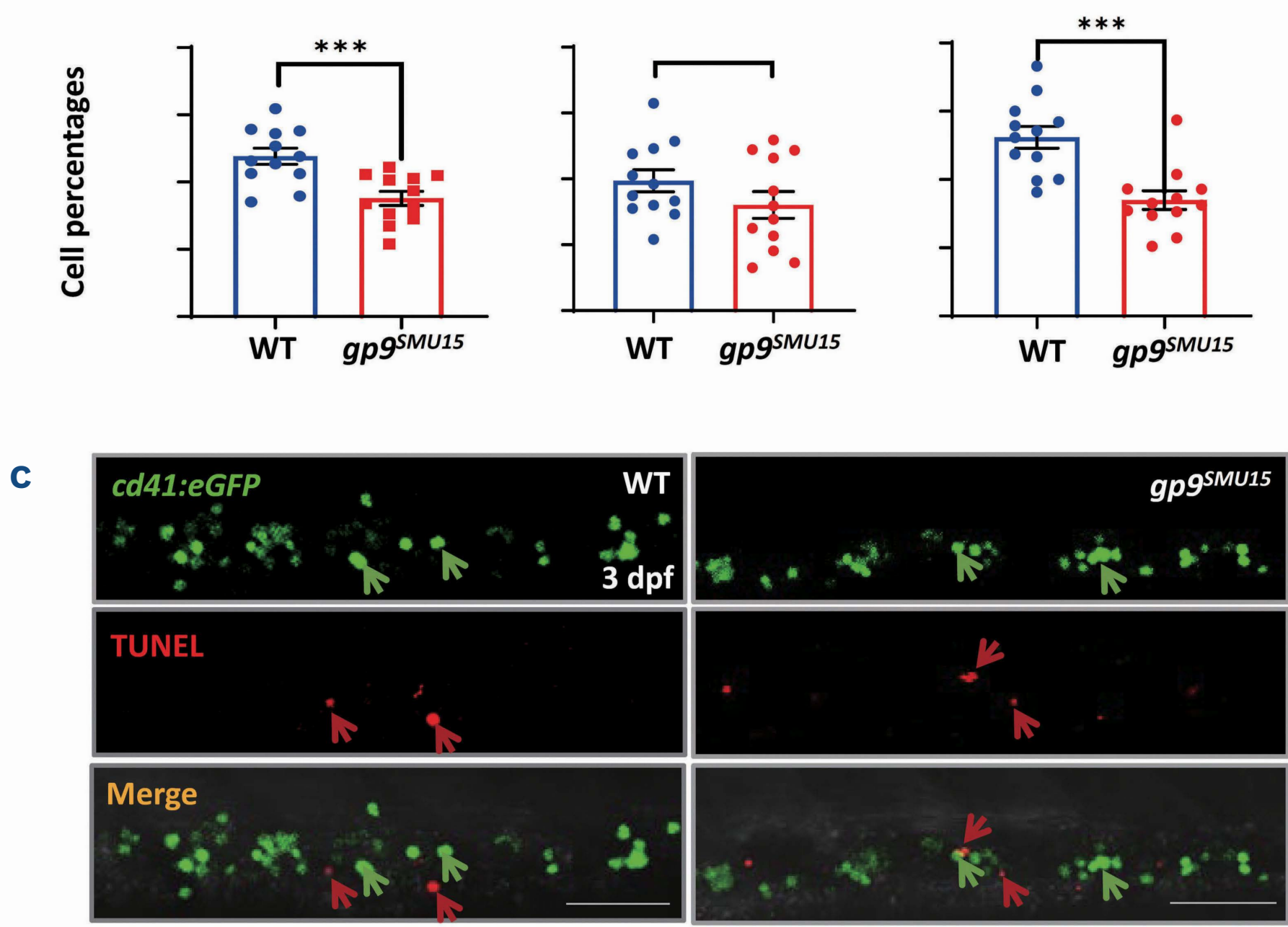

Figure 5. Abated thrombocyte precursor proliferation in gp9smu15 mutants. (A) Profiling of BrdU incorporation by caudal

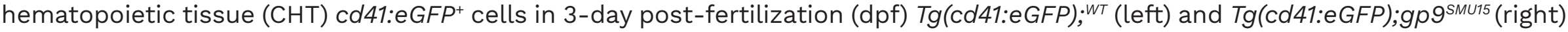
by double antibody staining of BrdU (red signals) and GFP (green signals). White triangular arrowheads indicate the yellow color merged signals represent the $B r d U^{+} c d 41^{\text {low }}$ cells. Gray triangular arrowheads indicate the yellow color merged signals represent the $B r d U^{+} c d 41^{\text {hiigh }}$ cells. The images of the stained samples were captured by setting the confocal parameter as pinhole size $35 \mu \mathrm{m}$ and 488 laser gain 700 to filter most cd41:eGFPlow signals, and the cd41:eGFPhigh thrombocytes fluorescent signals were counted. Scale bars: $50 \mu \mathrm{m}$. (B) Statistical data showing the percentages of CHT-localized cd41:eGFP ${ }^{+}$cells that incorporated BrdU. Asterisks indicate statistical difference, determined using a two-sample Student $t$-test, $n \geq 10$, mean \pm standard error of mean, ${ }^{*} P<0.01$. (C) No TUNEL incorporation with 3-dpf cd41:eGFP ${ }^{+}$cells in the CHT of Tg(cd41:eGFP);gp9s $9^{\text {SMU15 }}$ mutants (right panels) and their wild-type (WT) siblings (left panels). Green: GFP; red: TUNEL. The green arrowheads indicate the cd41:eGFP ${ }^{+}$cells and the red arrowheads indicate the TUNEL+ cells, No cd41:eGFP+TUNEL ${ }^{+}$cells were detected. Scale bars: $50 \mu \mathrm{m}$. 
in gp9sMU15 zebrafish embryos to evaluate the function of the two isoforms on thrombocytopoiesis. Unlike hsGP9wT overexpression, $h s^{\mathrm{GP} 970 \mathrm{~T}>\mathrm{C}}$ and $h s^{\mathrm{GP} 9182 \mathrm{~A}>\mathrm{G}}$ overexpression did not restore cd41:eGFPhigh thrombocyte counts in gp9sMu15 mutants (Figure 6B, C), suggesting that two human GP9 mutations affect GP9 function on thrombocytopoiesis. The above data demonstrate that the gp9 $9^{\text {SMU15 }}$ zebrafish model could serve as a good BSS model for functional validation of human GP9 mutations, and that $h S G P 9^{70 T>C}$ and hsGP ${ }^{182 A>G}$ are both loss-of-function mutations.

\section{Thrombopoietin and decitabine effectively modify} Bernard-Soulier syndrome phenotypes in the model In clinical settings, the primary treatment for BSS is bone marrow transplantation. Since BSS patients show symp- toms of thrombocytopenia, we wondered whether drugs clinically used for the treatment of thrombocytopenia would be effective to relieve BSS. We therefore utilized the Tg(cd41:eGFP);gp $9^{\text {SMU15 }}$ BSS zebrafish to test drug responses. rhTPO is a commercially available thrombocytopoietic agent approved by the US Food and Drug Administration (FDA) for the treatment of human thrombocytopenia. ${ }^{39}$ We treated 1.5-dpf WT sibling embryos with rhTPO until $4 \mathrm{dpf}$ to see whether zebrafish thrombocytes could respond to rhTPO (Figure $7 \mathrm{~A}$ ) and found that $100 \mathrm{U} / \mathrm{mL}$ rhTPO could effectively increase cd41:eGFPhigh thrombocyte numbers in both WT sibling embryos and gp9-deficient mutants. This suggested a conserved response from zebrafish thrombocytes to mammalian cytokines, and that rhTPO could relieve thrombocytopenia in BSS fish (Figure 7B, C).
A

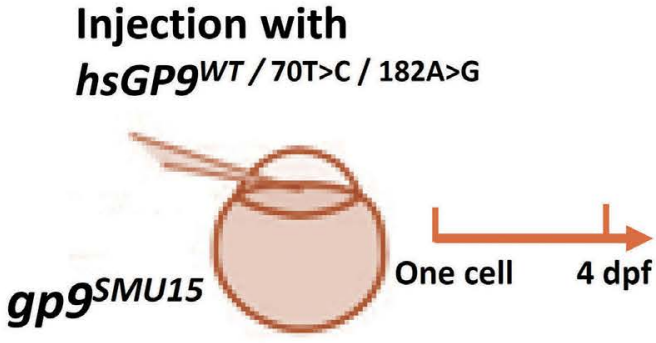

B

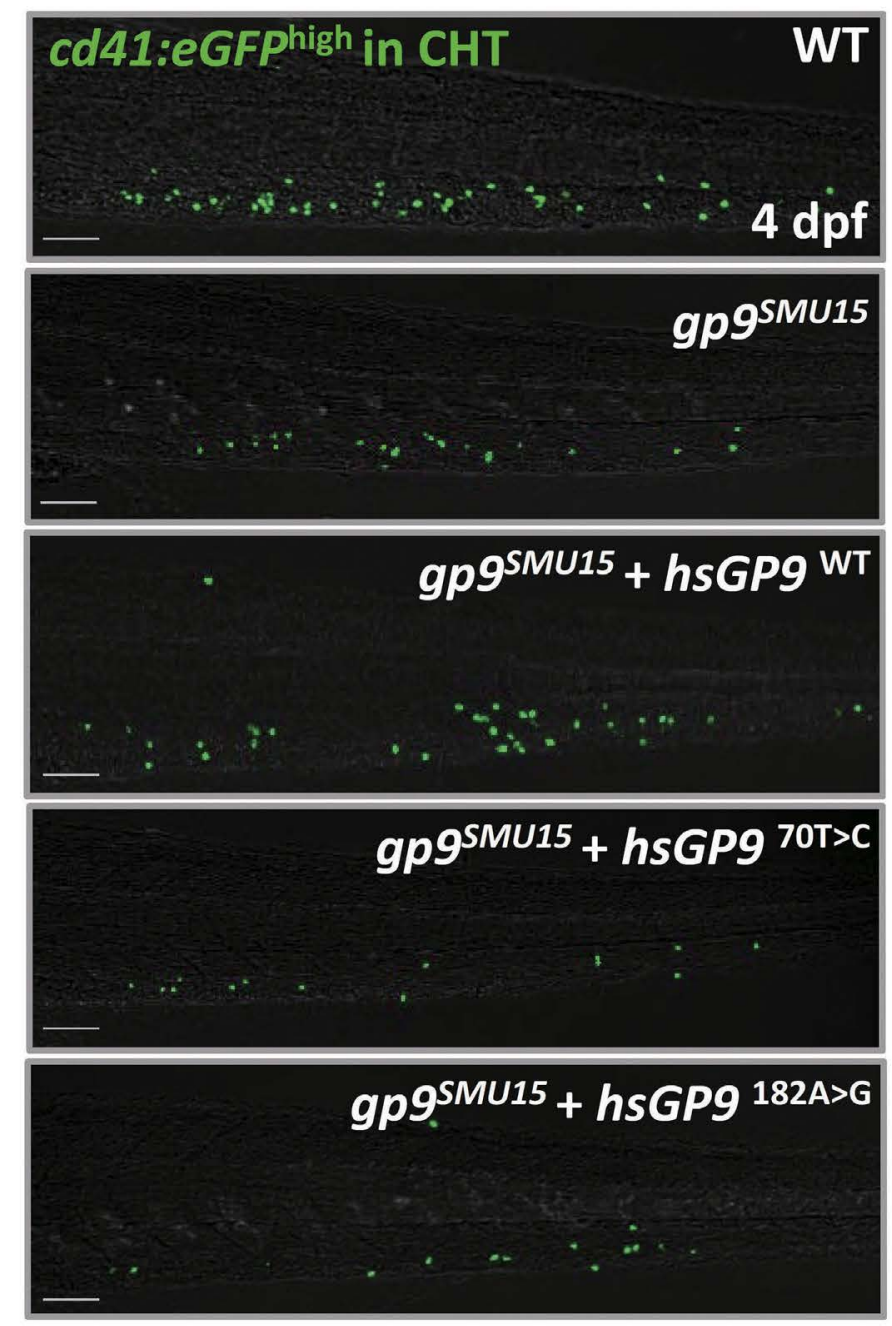

C

\section{cd41:eGFPhigh cell numbers in $\mathrm{CHT}$}

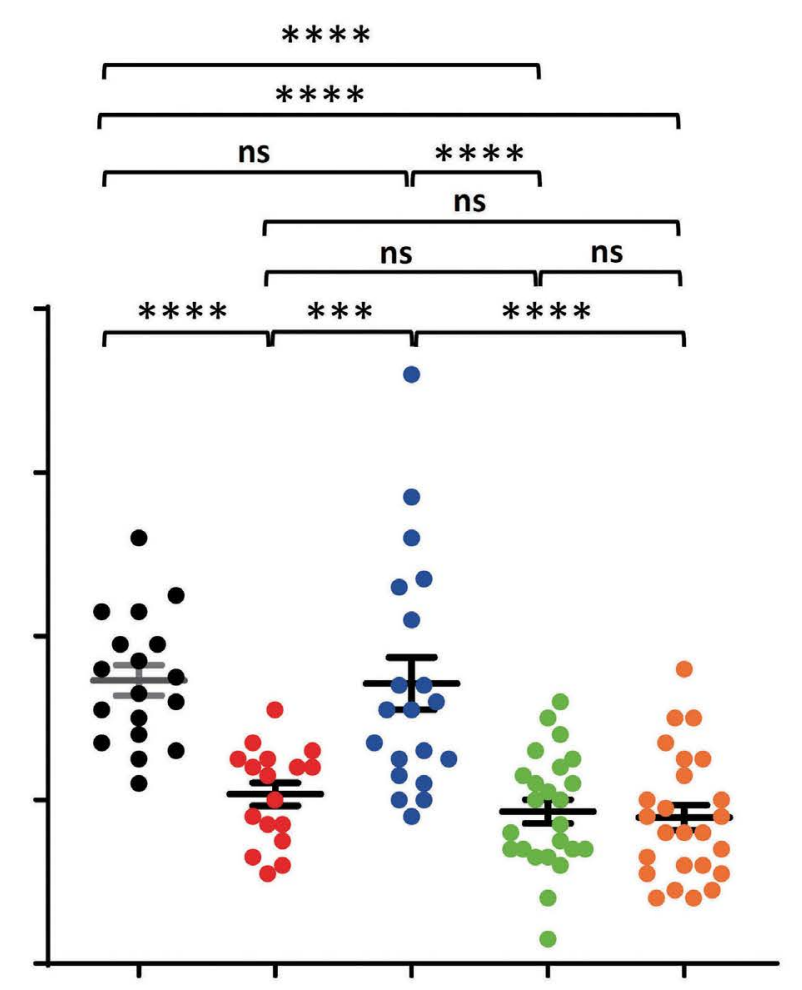

- WT

- $g$ p $9^{S M U 15}+h s G P 9^{70 T>C} \bigcirc g p 9^{S M U 15}+h s G P 9^{182 A>G}$

Figure 6. Functional validations of the human-derived GP9 mutations associated with Berndard-Soulier syndrome in the gp9 $9^{\text {smu15 }}$ zebrafish model. (A) Schematic diagram of the validation of the Bernard-Soulier syndrome (BSS) zebrafish model using human BSS-related GP9 mutations. hsGP9WT, hsGP970T>C and hsGP9182A>G mRNA were injected into one cell of the Tg(cd41:eGFP);gp9sMU15 embryos. (B) Representative images of staining of cd41:eGFP protein in 4 days post-fertilization (dpf) Tg(cd41:eGFP);WT and mutant Tg(cd41:eGFP);gp9sMu15 embryos. Images show GFPhigh signals. (C) Quantification of GFPhigh cell numbers at the caudal hematopoietic tissue $(\mathrm{CHT})$ region. Statistical significance was determined by a Student $t$-test, $\mathrm{n}>10$, mean \pm SEM, $* * * P<0.001, * * * * P<0.0001$. Scale bars: $50 \mu \mathrm{m}$. 
Decitabine, approved by the FDA for the treatment of leukemia and myelodysplastic syndrome, ${ }^{36}$ is now being used for the treatment of idiopathic thrombocytopenia purpura in clinical trials. ${ }^{37}$ Whether it could be used to treat BSS is unknown. We found that $20 \mu \mathrm{M}$ decitabine could effectively increase cd41:eGFPhigh thrombocytes in WT sibling embryos, and also expand thrombocytes in gp9 $9^{\text {sMu15 }}$ embryos (Figure 7D, E), suggesting that decitabine

A

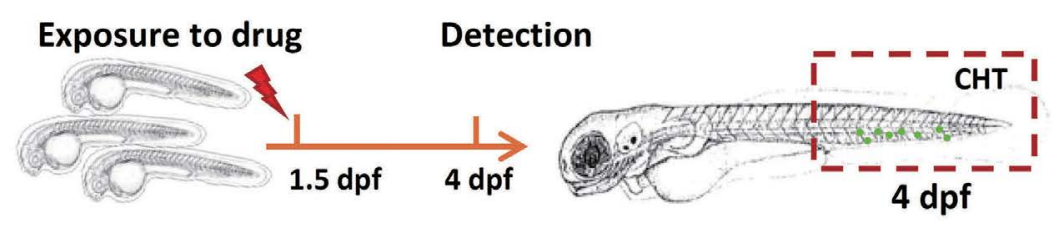

B
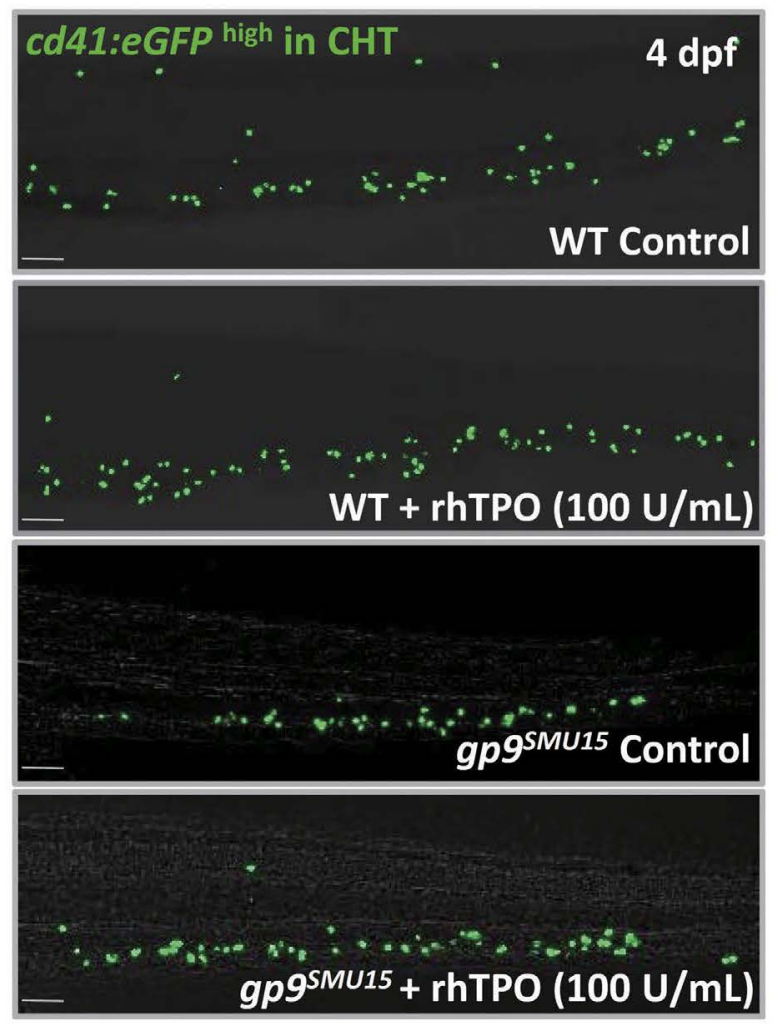

D
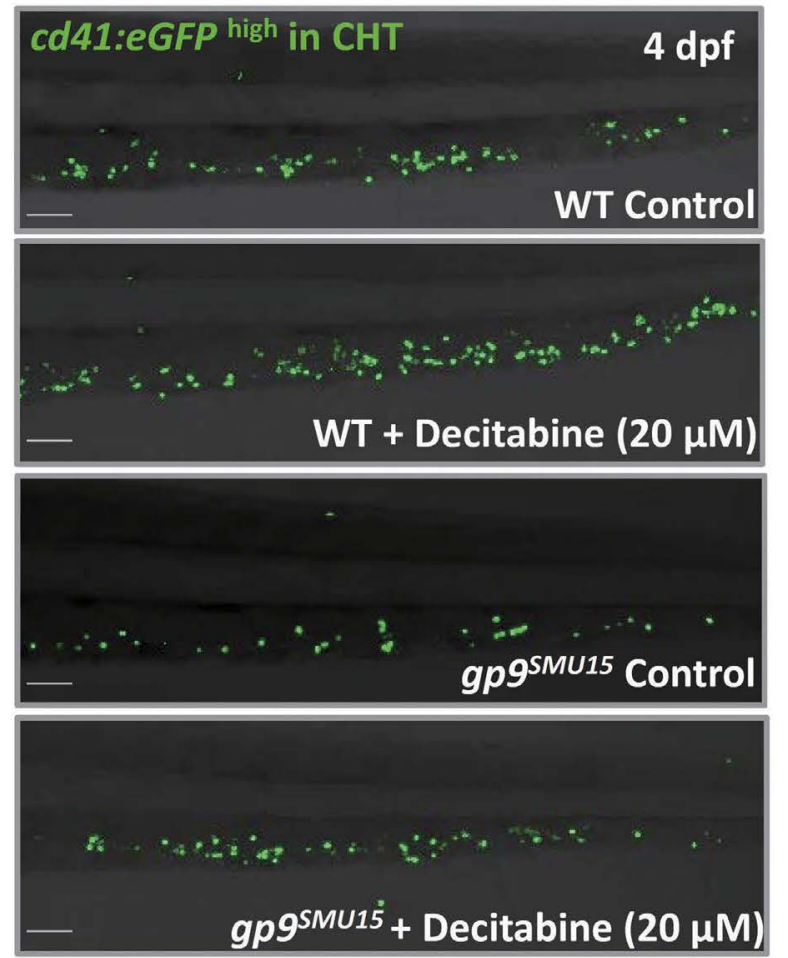

C cd41:eGFPhigh cell numbers in $\mathrm{CHT}$

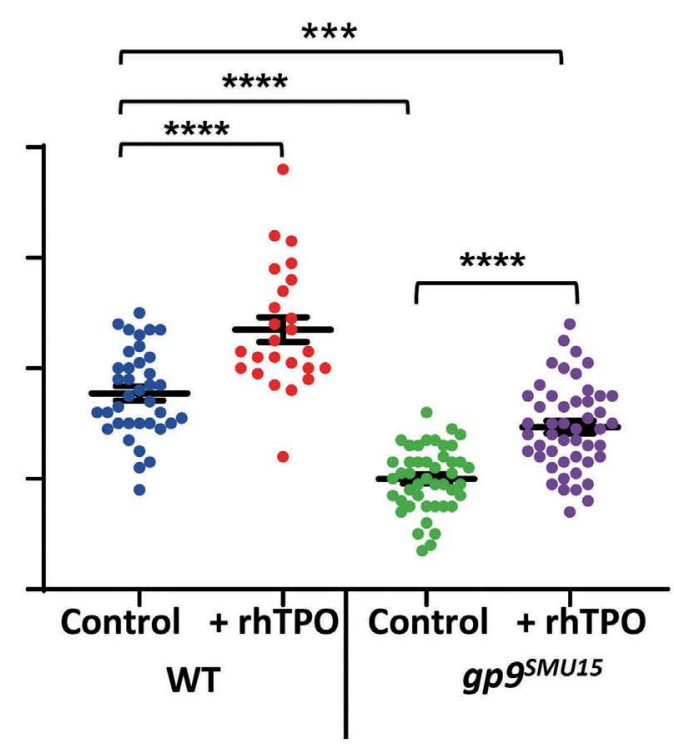

E cd41:eGFPhigh cell numbers in $\mathrm{CHT}$

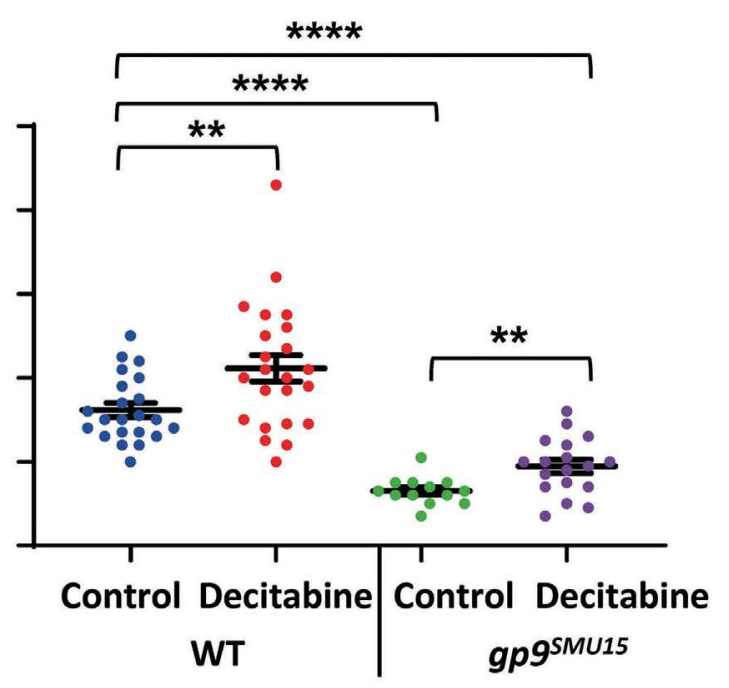

Figure 7. Therapeutic responses of Tg(cd41:eGFP);gp9smu15. (A) Schematic diagram of the drug treatment. The embryos were exposed to recombinant human thrombopoietin (rhTPO) and decitabine at 1.5 days post-fertilization (dpf) and fixed at 4 dpf. (B) Thrombocytopenia indicated by decreased cd41:eGFPhigh cells in Tg(cd41:eGFP);gp9smu15 mutant embryos and thrombocyte expansion by rhTPO. Images show GFPhigh signals. Representative images of Tg(cd41:eGFP);WT and Tg(cd41:eGFP); 9 g $^{\text {SMU15 }}$ embryos treated with sodium chloride saline control and rhTPO (B). (C) Statistical significance was determined using a paired Student ttest, $\mathrm{n} \geq 5$, mean \pm standard error of mean (SEM), $* P<0.05, * \star P<0.01, * \star \star P<0.001, * \star \star \star * P<.0001$. (D) Representative images of Tg(cd41:eGFP);WT and Tg(cd41:eGFP);gp9smu15 embryos treated with dimethylsulfoxide (DMSO) control and decitabine. Images show GFPhigh signals. (E) Quantification of cd41:eGFPhigh numbers in the caudal hematopoietic tissue (CHT). Statistical significance was determined by a paired Student $t$-test, $\mathrm{n}>10$, mean \pm SEM. ${ }^{*} P<0.05,{ }^{*} * P<0.01, * \star * P<0.001,{ }^{*} * \star * P<0.0001$. Scale bars: $50 \mu \mathrm{m}$. 
could increase the number of zebrafish thrombocytes and may relieve thrombocytopenia in the BSS model. The above results demonstrate that both rhTPO and decitabine could effectively relieve thrombocytopenia in BSS zebrafish (although thrombocyte numbers were not fully rescued), suggesting that these drugs, clinically used for thrombocytopenia, could also be applied in the treatment of BSS. The response of the BSS zebrafish model to these therapeutic agents suggests that the model could be useful for the evaluation and screening of thrombopoietic drugs.

\section{Discussion}

BSS, characterized by prolonged bleeding times, enlarged platelets, an inability to clot and thrombocytopenia, are bleeding disorders that result from mutations in genes of the glycoprotein Ib complex, including GP1bA, GP1bb and GP9.10 GP9 in particular is the most frequently mutated gene reported in a group of BSS patients from 211 families. ${ }^{10}$ In this study, we generated and characterized a CRISPR-Cas9 targeted gp $9^{\text {sMu15 }}$ zebrafish mutant, which is the first established inherited BSS zebrafish model. The gp9-deficient zebrafish were viable and displayed thrombocytopenia, with a bleeding disorder, thus resembling Gp9-knockout mice and human BSS patients. The gp9 ${ }^{\text {SMu15 }}$ zebrafish mutant provides a complementary model to aid our understanding of the various roles of gp9 in thrombocytopoiesis and in BSS. Thrombocytopenia is an important feature reported in both human BSS and Gp9 knockout mice. Our data strengthened our understanding of the role of gp9 in thrombocytopoiesis in vertebrates, as gp9-deficient zebrafish showed a consistent thrombocytopenia phenotype from embryonic stages to adulthood. We also demonstrated that gp9smu15 fish showed reduced thrombocytes but increased precursor cells from embryonic to adult stages. Interestingly, the $3 \mathrm{dpf}$ whole-mount in situ hybridization showed unaltered cmyb expression but cd41:eGFPlow cells were increased, suggesting that the increased col41:eGFPlow cells were likely thrombocyte-erythroid progenitors rather than $c m y b^{+}$ hematopoietic stem cells or other lineage progenitors. In particular, their KM cd41:eGFP ${ }^{+}$cells were larger than their siblings, similar to the larger thrombocytic cell volumes in mice and humans. ${ }^{9,16}$ As regards the cellular mechanisms for the thrombocytopenia caused by gp9 mutations, we further clarified that thrombocytopenia may be attributed to proliferation defects in thrombocyte lineage cells. It is probable that proliferation blocked precursors or thrombocyte progenitors had consequent problems in differentiating into thrombocytes.

Thrombocytes in zebrafish are the functional equivalent of mammalian platelets, playing major roles in clotting and preventing bleeding. ${ }^{18}$ Patients with thrombocytopenia or bleeding disorders can have severe hemorrhage following injury or surgery. Similarly to BSS patients, gp9-deficient zebrafish also display a spontaneous bleeding syndrome, accompanied by a prolonged bleeding time after injury. Taken together, these results confirm that gp9 deficiency increases the tendency to bleeding, which is conserved from zebrafish to humans. The bleeding disorders in gp9deficient animals and patients could be attributed to insufficiently functional thrombocytes. The activation of thrombocytes may also be affected in GP9-mutated individuals, according to several reports. ${ }^{38,39}$ Taken together, these results suggested that zebrafish with inherited gp9deficiency, displaying a bleeding disorder resembling human BSS, could serve as a complementary model to broaden our understanding of the various roles of GP9 in thrombocytopoiesis, as well as in thrombosis and hemostasis.

Based on the conservation of thrombocytopoiesis regulatory factors and strong similarity of GPIX across species, we verified that human GP9 could compensate zebrafish gp9, as human GP9WT mRNA is able to rescue thrombocytopenia in gp $9^{\text {sMu15 }}$ zebrafish. For functional validation of the BSS patient-derived mutations, previous studies were mainly restricted to cell lines. ${ }^{40,41}$ Here we provide an example of fast verification of patient-derived mutations using zebrafish, as we showed that gp $9^{\text {sMu15 }}$ zebrafish could serve as a cost-effective model for validating human GP9 mutations associated with BSS. The two GP9 mutations ( $h s G P 9^{70 T>C}$ and $h s G P 9^{182 A>G}$ ) associated with BSS were evaluated by simply overexpressing them in the zebrafish model, and the failure to rescue thrombocytopenia confirmed that the two mutations are loss-of-function mutations in thrombocytopoiesis. Thus, the BSS zebrafish model with a high-throughput screening capability could help to accelerate the discovery of potential genetic variants of unknown clinical significance in vivo.

The primary clinical treatment for BSS is supportive with platelet transfusions, anti-fibrinolytics particularly for mucocutaneous bleeds and recombinant activated factor VII in attempts to shorten bleeding times. ${ }^{42-44}$ More effective ways to treat BSS are much needed. Previous studies have shown that rhTPO rapidly increases platelet counts in patients with idiopathic thrombocytopenia purpura, ${ }^{45}$ and lowdose decitabine promotes thrombocytopoiesis in idiopathic thrombocytopenia purpura and healthy controls. ${ }^{46} \mathrm{Here}$, we showed that rhTPO and decitabine could efficiently relieve BSS phenotypes in zebrafish larvae (Figure 7). Given that the thrombocytopenia deficiency could be consistently observed in both larval and adult stages of zebrafish (Figures 2 and 4), we think that the two drugs may still relieve thrombocytopenia in adult BSS zebrafish if administered at a proper dose using a suitable way of drug delivery. The BSS zebrafish model will be beneficial for drug discovery, as evidenced by the demonstration that rhTPO and decitabine, 
used for promoting thrombocytopoiesis, also effectively relieved BSS phenotypes in the zebrafish larvae, which may also shed light on the treatment of BSS by promoting thrombocytopoiesis.

In summary, we established a gp9-deficient zebrafish model displaying thrombocytopenia and a bleeding disorder, which resemble the clinical features of BSS in patients. We confirmed the conservation of the roles of GP9 in thrombocytopoiesis as well as in thrombosis and hemostasis from zebrafish to mammals. The BSS zebrafish model could be utilized as a cost-effective model to evaluate the function of human related mutations, and this line may also be applied in in vivo screening for novel drugs to treat BSS. The zebrafish BSS model, as a proofof-principle example, together with other established thrombocyte related zebrafish models, will allow us to examine the effect of clinically discovered mutations with uncertain clinical significance in thrombocyte development and function, as well as to conduct a robust drug evaluation or establish a screening platform to assess the therapeutic response of BSS, particularly against mutations with thrombocytopenia.

\section{Disclosures}

No conflicts of interest to disclose.

\section{Contributions}

$Y Z$ conceived and supervised the study and wrote, reviewed and edited the article; QL conceived the study, performed investigations and wrote, reviewed and edited the article; $R Z$ and $P M$ performed investigations, wrote, reviewed and edited the article; $L W, L Y, W L, J W, Y C$ and $L S$ performed investigations, analyzed the data and wrote the original draft of the manuscript.

\section{Acknowledgments}

The authors thank Dr. Jin Xu for his constructive suggestions and Mr. Xiaohui Chen for helping with the maintenance of zebrafish lines.

\section{Funding}

This work was supported by the National Natural Science Foundation of China (81870100 and 31871475), the National Key R\&D Program of China (2018YFA0800200), Guangdong Province Universities and Colleges Pearl River Scholar Funded Scheme (2019), and Fundamental Research Funds for the Central Universities (2019ZD54 and 2018MS69).

\section{Data-sharing statement}

The data supporting the findings of this study are available within the article and its supplementary material.

\section{References}

1. Nakao K, Angrist AA. Membrane surface specialization of blood platelet and megakaryocyte. Nature. 1968;217(5132):960-961.

2. Lordier L, Jalil A, Aurade F, et al. Megakaryocyte endomitosis is a failure of late cytokinesis related to defects in the contractile ring and Rho/Rock signaling. Blood. 2008;112(8):3164-3174.

3. Machlus KR, Italiano JJ. The incredible journey: from megakaryocyte development to platelet formation. J Cell Biol. 2013; 201(6):785-796.

4. Woolthuis CM, Park CY. Hematopoietic stem/progenitor cell commitment to the megakaryocyte lineage. Blood. 2016;127(10):1242-1248.

5. Bianchi E, Norfo R, Pennucci V, Zini R, Manfredini R. Genomic landscape of megakaryopoiesis and platelet function defects. Blood. 2016;127(10):1249.

6. Lanza F. Bernard-Soulier syndrome (hemorrhagiparous thrombocytic dystrophy). Orphanet J Rare Dis. 2006;1:46.

7. Bernard J, Soulier JP. Sur une nouvelle variété de dystrophie thrombocytaire-hémorragipare congénitale. Sem Hop Paris. 1948;24(Spec. No.):3217-3222

8. de la Salle C, Lanza F, Cazenave JP. Biochemical and molecular basis of Bernard-Soulier syndrome: a review. Nouv Rev Fr Hematol. 1995;37(4):215.

9. Lopez J, Andrews R, Afshar-Kharghan V, Berndt M. BernardSoulier syndrome. Blood. 1998;91:4397-4418.

10. Savoia A, Kunishima S, De Rocco D, et al. Spectrum of the mutations in Bernard-Soulier syndrome. Hum Mutat. 2014;35(9):1033-1045.

11. McEwan PA, Yang W, Carr KH, et al. Quaternary organization of GPIb-IX complex and insights into Bernard-Soulier syndrome revealed by the structures of GPIb $\beta$ and a GPIb $\beta /$ GPIX chimera.
Blood. 2011;118(19):5292-5301

12. Clemetson JM, Kyrle PA, Brenner B, Clemetson KJ. Variant Bernard-Soulier syndrome associated with a homozygous mutation in the leucine-rich domain of glycoprotein IX. Blood. 1994;84(4):1124-1131.

13. Rivera CE, Villagra J, Riordan M, Williams S, Lindstrom KJ, Rick $M E$. Identification of a new mutation in platelet glycoprotein IX (GPIX) in a patient with Bernard-Soulier syndrome. Br J Haematol. 2001;112(1):105-108.

14. Dağıstan N, Kunishima S. First Turkish case of Bernard-Soulier syndrome associated with GPIX N45S. Acta Haematol. 2007;118(3):146-148.

15. Ali S, Ghosh K, Shetty S. Novel genetic abnormalities in Bernard-Soulier syndrome in India. Ann Hematol. 2014;93(3):381-384.

16. Meehan TF, Conte N, West DB, et al. Disease model discovery from 3,328 gene knockouts by The International Mouse Phenotyping Consortium. Nat Genet. 2017; 49(8):1231-1238.

17. Jagadeeswaran P, Liu YC, Sheehan JP. Analysis of hemostasis in the zebrafish. Methods Cell Biol. 1999;59:337-357.

18. Jagadeeswaran P, Sheehan JP, Craig FE, Troyer D. Identification and characterization of zebrafish thrombocytes. $\mathrm{Br} \mathrm{J}$ Haematol. 1999;107(4):731-738.

19. Lin H-F, Traver D, Zhu H, et al. Analysis of thrombocyte development in CD41-GFP transgenic zebrafish. Blood. 2005;106(12):3803-3810.

20. Khandekar G, Kim S, Jagadeeswaran P. Zebrafish thrombocytes: functions and origins. Adv Hematol. 2012;2012:857058.

21. Lin Q, Zhang $Y$, Zhou R, et al. Establishment of a congenital amegakaryocytic thrombocytopenia model and a thrombocyte- 
specific reporter line in zebrafish. Leukemia. 2017;31(5):1206-1216.

22. Rost MS, Shestopalov I, Liu Y, et al. Nfe2 is dispensable for early but required for adult thrombocyte formation and function in zebrafish. Blood Adv. 2018;2(23):3418-3427.

23. Marconi C, Di Buduo CA, LeVine K, et al. Loss-of-function mutations in PTPRJ cause a new form of inherited thrombocytopenia. Blood. 2019;133(12):1346-1357.

24. Ma AC, Cheung AM, Ward AC, et al. The study of Jak2 V617F mutation in polycythemia vera with zebrafish model. Cell Res. 2008;18(S1):S141-S141.

25. Albers CA, Cvejic A, Favier R, et al. Exome sequencing identifies NBEAL2 as the causative gene for gray platelet syndrome. Nat Genet. 2011;43(8):735-737.

26. Gregory M, Hanumanthaiah R, Jagadeeswaran P. Genetic analysis of hemostasis and thrombosis using vascular occlusion. Blood Cells Mol Dis. 2002;29(3):286-295.

27. Kim S, Carrillo M, Kulkarni V, Jagadeeswaran P. Evolution of primary hemostasis in early vertebrates. PLoS One. 2009;4(12):e8403.

28. Deebani A, lyer N, Raman R, Jagadeeswaran P. Effect of MS222 on hemostasis in zebrafish. J Am Assoc Lab Anim Sci. 2019;58(3):390-396.

29. Zheng L, Abdelgawwad MS, Zhang D, et al. Histone-induced thrombotic thrombocytopenic purpura in adamts $13^{-/-}$zebrafish depends on von Willebrand factor. Haematologica. 2020;105(4):1107-1119.

30. Macaulay IC, Svensson V, Labalette C, et al. Single-cell RNAsequencing reveals a continuous spectrum of differentiation in hematopoietic Cells. Cell Rep. 2016;14(4):966-977.

31. Ma D, Zhang J, Lin HF, Italiano J, Handin RI. The identification and characterization of zebrafish hematopoietic stem cells. Blood. 2011;118(2):289-297.

32. Svoboda O, Stachura DL, Machoňová O, et al. Dissection of vertebrate hematopoiesis using zebrafish thrombopoietin. Blood. 2014;124(2):220-228.

33. Savoia A, Pastore A, De Rocco D, et al. Clinical and genetic aspects of Bernard-Soulier syndrome: searching for genotype/phenotype correlations. Haematologica. 2011;96(3):417-423.

34. Kissa K, Murayama E, Zapata A, et al. Live imaging of emerging hematopoietic stem cells and early thymus colonization. Blood. 2008;111(3):1147-1156.
35. Mo X, Nguyen NX, Mcewan PA, et al. Binding of platelet glycoprotein $\operatorname{lb} \beta$ through the convex surface of leucine-rich repeats domain of glycoprotein IX. J Thromb Haemost. 2009;7(9):1533-1540.

36. Jabbour E, Issa JP, Garcia-Manero G, Kantarjian H. Evolution of decitabine development: accomplishments, ongoing investigations, and future strategies. Cancer. 2008;112(11):2341-2351.

37. Zhou H, Qin P, Liu Q, et al. A prospective, multicenter study of low dose decitabine in adult patients with refractory immune thrombocytopenia. Am J Hematol. 2019;94(12):1374-1381.

38. Michelson AD, Benoit SE, Furman MI, Barnard MR, Nurden P, Nurden AT. The platelet surface expression of glycoprotein $V$ is regulated by two independent mechanisms: proteolysis and a reversible cytoskeletal- mediated redistribution to the surface-connected canalicular system. Blood. 1996;87(4):1396-1408.

39. Canobbio I, Balduini C, Torti M. Signalling through the platelet glycoprotein Ib-V-IX complex. Cell Signal. 2004;16(12):1329-1344.

40. Zmajkovic J, Lundberg P, Nienhold R, et al. A gain-of-function mutation in EPO in familial erythrocytosis. N Engl $J$ Med. 2018;378(10):924-930.

41. Kim AR, Ulirsch JC, Wilmes $\mathrm{S}$, et al. Functional selectivity in cytokine signaling revealed through a pathogenic EPO mutation. Cell. 2017;168(6):1053.

42. Grainger JD, Thachil J, Will AM. How we treat the platelet glycoprotein defects; Glanzmann thrombasthenia and Bernard Soulier syndrome in children and adults. $\mathrm{Br} J$ Haematol. 2018;182(5):621-632.

43. Almeida AM, Khair K, Hann I, Liesner R. The use of recombinant factor VIla in children with inherited platelet function disorders. Br J Haematol. 2003;121(3):477-481.

44. Ozelo MC, Svirin P, Larina L. Use of recombinant factor VIla in the management of severe bleeding episodes in patients with Bernard-Soulier syndrome. Ann Hematol. 2005;84(12):816-822.

45. Wang $S$, Yang R, Zou P, et al. A multicenter randomized controlled trial of recombinant human thrombopoietin treatment in patients with primary immune thrombocytopenia. Int J Hematol. 2012;96(2):222-228.

46. Zhou H, Hou Y, Liu X, et al. Low-dose decitabine promotes megakaryocyte maturation and platelet production in healthy controls and immune thrombocytopenia. Thromb Haemost. 2015;113(5):1021-1034. 